[3] J. Górniak, Dilations of positive definite operator valued functions in linear spaces (in Polish), Komunikaty Inst. Mat. Polit. Wrock. Nr 73 (1977). [See also: Locally convex spaces with the factorization property, Colloq. Math. (to appear).]

[4] - Remarks on positive definite operator valued functions in linear spaces, Lecture Notes in Math., vol. 656, Springer Verlag, 1978, 37-44.

[5] - An analogue of Sz.-Nagy's dilation theorem II, Bull. Acad. Polon. Sci., Sér. Sci. Math. Astrononom. Phys. 27 (1979), 287-289.

[6] J. Górniak and A. Weron, An analogue of $S z .-N a g y ' s$ dilation theorem, ibid. 24 (10) (1976), 867-872.

[7] A. N. Kolmogorov, Stationary sequences in Hilbert space (in Russian), Bull. Math. Univ. Moscow 2 (1941), 1-40.

[8] D. Kölzow, A survey of Abstract Wiener Spaces (preprint, 1974).

[9] P. Masani, An explioit treatment of dilation theory (preprint, Autumn 1975). [See also an enlarged version: Dilations as propagators of Hilbertian varieties, SIAM J. Math. Analysis 9 (1978), 414-456.]

[10] - Propagators and dilations, Lecture Notes in Math. vol. 656, Springer Verlag, $1978,95-117$.

[11] W. MIak, Dilations of Hilbert space operators (General theory), Dissertationes Math. 158 (1978), 1-61.

[12] W. Mlak and A. Weron, Dilations of Banach spaces operator valued functions, Ann. Polon. Math. 38 (1980), 295-303.

[13] N. N. Vakhania, Probability distributions on linear spaces (in Russian), Mecniereba, Tbilisi 1971. [English edition: North-Holland, 1981.]

[14] N. N. Vakhania and V. I. Tarieladze, Covariance operators of probability measures in locally convex spaces (in Russian), Teor. Verojatnost. i Primenen. 23 (1) (1978), 3-26.

[15] A. Weron, Prediction theory in Banach spaces, Lecture Notes in Math., vol. 472, Springer Verlag, 1975, 207-228.

[16] - Second order stochastic processes and dilation theory in Banach spaces, Ann. Inst. H. Poincaré 16 (1980), 29-38.

[17] - Remarles on positive-definite operator valued functions in Banach spaces, Bull. Acad. Polon. Sci. 24 (10) (1976), 873-876.

INSTITUTE OF MATHEMATIOS

WROCEAW TTCGHICAL UNTVIRSITY

50-370, WROCF AW, POTAND
Received May 24, 1978

in revised form August 30,1978

\section{Convexity, type and the three space problem}

by

\section{N. J. KALTON (Columbia, Mo.)*}

Abstract. A twisted sum of two quasi-Banach spaces $X$ and $Y$ is a quasi-Banach space $Z$ with a closed subspace $X_{0} \cong X$ such that $Z / X_{0} \cong Y$.

We show that if $X$ is $p$-convex and $Y$ is $q$-convex where $p \neq q$, then $Z$ is $\min (p q)$ convex. Similarly, if $X$ is a type $p$ Banach space and $Y$ is a type $q$ Banach space where $p \neq q$ then $Z$ is type $\min (p, q)$.

If $X$ and $\bar{Y}$ are Banach spaces, we show that $Z$ is $\log$ convex, i.e., for some $O<\infty$

$$
\left\|z_{1}+\ldots+z_{n}\right\| \leqslant C\left(\sum_{k=1}^{n}\left\|z_{k}\right\|\left(1+\log \frac{1}{\left\|z_{z_{e}}\right\|}\right)\right)
$$

where $\left\|z_{1}\right\|+\ldots+\left\|z_{n}\right\|=1$. Conversely, every log convex space is the quotient of a subspace of a twisted sum of two Banach spaces.

If $X$ and $Y$ are type $p$ Banach spaces $(1<p \leqslant 2)$ and one is the quotient of a subspace of some $L_{p}$-space, then $Z$ is log type $p$, i.e.,

$$
\left\{\int_{0}^{1}\left\|\varepsilon_{1}(t) z_{1}+\cdots+\varepsilon_{n}(t) z_{n}\right\|^{p} d t\right\}^{1 / p} \leqslant c\left\{\sum\left\|z_{z_{k}}\right\|^{p}\left(1+\left(\log \frac{1}{\left\|z_{k_{k}}\right\|}\right)^{p}\right)\right\}^{1 / p} .
$$

where $\left\|z_{1}\right\|^{p}+\ldots+\left\|z_{n}\right\|^{p}=1$. This result is best possible in a certain sense.

We also show that if $p<1$ type $p$ implies $p$-convexity, but if $p=1$ a,type 1 space need not be convex.

We investigate which Orlicz sequence spaces and Köthe sequence spaces are $\mathscr{K}$-spaces, i.e., such that every twisted sum with $\boldsymbol{R}$ is a direct sum.

1. Introduction. A quasi-Banach space $Z$ is a twisted sum of $X$ and $Y$ if it has a subspace $X_{0} \cong X$ such that $Z / X_{0} \cong Y$. The so-called three space problem is to study the properties of $Z$ in terms of those of $X$ and $Y$.

In [1], Enflo, Lindenstrauss and Pisier showed that a Banach space which is a twisted sum of two Hilbert spaces need not be a Hilbert space. Independently, the author [6], Ribe [15] and Roberts [16] showed that a twisted sum of a line and a Banach space need not be locally convex. In [9] the author and. Peck showed that these results are related by describing a general construction which shows that for every $p, 0<p<\infty$, there is a twisted sum of $l_{p}$ with $l_{p}$ which is not a direct sum. In particular, for $0<p<1$, there is a non $p$-convex space which is a twisted sum of two $p$-convex spaces.

* University of Missouri. Research done while the author held visiting positions at Michigan State University and the University of Illinois. 
In contrast to these.negative results there are a number of theorems which say that a twisted sum cannot be too bad. In [1] the twisted sum of two type 2 Banach spaces is shown to be type $p$ for all $p<2$. In [6] it is shown that if $X$ is a Banach space and $Y$ is a type $p$ Banach space for some $p>1$, then a twisted sum of $X$ and $Y$ (in that order) is convex (i.e., a Banach space). Also if $X$ is $p$-convex $(0<p \leqslant 1$ ) and $Y$ is $q$-convex $(0<q<p)$, then any twisted sum of $X$ and $Y$ (in that order) is again $q$-convex.

These suggest a general principle, if we regard $p$-convexity $(0<p \leqslant 1)$ or type $p(0<p \leqslant 2)$ as an index of "roundness". The twisted sum of . two spaces of differing degrees of roundness will retain the properties of. the less round space; the twisted sum of two spaces of equal roundness may however be less round than either. The main aim of this paper is to establish such a pattern, and to examine more precisely the case of equal roundness.

First in Section 3, we introduce a new class of quasi-Banach spaces which we name logconvex. A space $X$ is log convex if either of the following two equivalent conditions holds for some $C, C^{*}<\infty$

$$
\left\|x_{1}+\ldots+x_{n}\right\| \leqslant C \sum_{i=1}^{n}\left\|x_{i}\right\|\left(1+\log \frac{1}{\left\|x_{i}^{\prime}\right\|}\right)
$$

wherever $\left\|x_{1}\right\|+\ldots+\left\|x_{n}\right\|=1, x_{1}, \ldots, x_{n} \in X$ or

$$
\left\|x_{1}+\ldots+x_{n}\right\| \leqslant C^{*} \sum_{j=1}^{n}\left\|x_{j}\right\|(1+\log j) .
$$

Logconvex spaces play an important role in this paper; they are, in a sense, the next best thing to being Banach spaces. An example is the space $I(1, \infty)$ (i.e., weak $L_{1}$ ).

In Section 4 we show that if $p<1$, type $p$ is equivalent to $p$-convexity, so that we reduce the study of type to the case $1 \leqslant p \leqslant 2$.

Section 5 contains some injtial technical results in twisted sums which contain very little that is new. Lemma 5.2 essentially reproduces a result of $[1]$ in rather more generality.

In Section 6 we show that if $X$ and $Y$ are $p$-convex and $q$-convex, respectively, where $p<q$, then any twisted sum of $X$ and $Y$ or of $Y$ and $X$ is $p$-convex. (One half of this result is in [6], see above). In a similar vein, if $X$ and $Y$ are type $p$ and type $q$, respectively, where $1 \leqslant p<q \leqslant 2$, then any twisted sum $Z$ of $X$ and $Y$ or $Y$ and $X$ is type $p$. Let us remark here that the methods of [1] (cf. also [13]) show that in either case if $z_{1}, \ldots, z_{n}$ $\in \mathbb{Z}$

$$
\left\{\int_{0}^{1}\left\|\sum \varepsilon_{i} z_{i}\right\|^{2} d t\right\}^{1 / 2} \leqslant C n^{1 / p-1 / 2}\left(\sum_{i=1}^{n}\left\|z_{i}\right\|^{2}\right)^{1 / 2}
$$

The main step in the argument here is to pass from this inequality to establishing type $p$ (but only for twisted sums where the other space is type $q>p$ ). As shown in [13] (1.0.3) implies type $r$ for $r<p$. We note that a type 1 space need not be convex.

In Section 7 we study the case $p=q$. We show that any twisted sum of two Banach spaces is logconvex, and this result is best possible. In fact, a space is logconvex if and only if it is a quotient of some subspace of a twisted sum of two Banach spaces. The corresponding results for type $p$ are right if we assume that one of the spaces $X$ and $Y$ is a quotient of a subspace of a space $L_{p}(\mu)$. In that case any twisted sum $Z$ is $\log$ type $p$, i.e.,

$$
\left\{\int_{0}^{1}\left\|\sum \varepsilon_{i} z_{i}\right\|^{p} d t\right\}^{1 / p} \leqslant C\left(1+\sum\left\|z_{i}\right\|^{p}\left(\log \frac{1}{\left\|z_{i}\right\|}\right)^{p}\right)^{1 / p}
$$

whenever $\left\|z_{1}\right\|^{p}+\ldots+\left\|z_{n}\right\|^{p}=1$, or equivalently

$$
\left\{\int_{0}^{1}\left\|\sum_{i=1}^{n} \varepsilon_{i} z_{i}\right\|^{p} d t\right\}^{1 / p} \leqslant C^{*}\left(\sum_{k=1}^{n}\left\|z_{k}\right\|^{p}(1+\log k)^{p}\right)^{1 / p}
$$

for $z_{1}, \ldots, z_{n} \in Z$. Furthermore this is best possible for the twisted sum of $l_{p}$ and $l_{p}(1 \leqslant p \leqslant 2)$ constructed in [9] contains a copy of the Orlicz space $l_{w}$ where

$$
\psi(t)=t^{p}\left[1+\left(\log \frac{1}{t}\right)^{p}\right]
$$

near zero, and for this space (1.0.4) cannot be improved.

In Section 8 we examine twisted sums of $\boldsymbol{R}$ and $l_{1}$ more closely, showing in particular that the examples in [6] and [15] are non-equivalent but both are best possible in a certain sense.

In Section 9 we classify those non-locally convex Orlicz spaces $l_{f} \subset l_{1}$ which are $\mathscr{K}$-spaces, i.e., for which every twisted sum of $\boldsymbol{R}$ and $l_{f}$ is a direct sum. In particular, this applied to examining "galb" conditions of the type $\left(\sum f\left(\left\|x_{i}\right\|\right)<\infty \Rightarrow \sum x_{i}\right.$ converges) which are preserved under twisted sums with $\boldsymbol{R}$. It is shown that if $f$ is submultiplicative, this condition will be preserved if and only if $f(x) \geqslant c x^{p}$ for some $p<1$.

In Section 10, we examine those locally convex $F$-spaces $X$ which are not locally bounded, but are $\mathscr{K}$-spaces, so that they have the property that if $Y$ is locally convex any twisted sum of $Y$ and $X$ is locally convex. It is shown that every nuclear space is a $\mathscr{K}$-space, and Köthe spaces which are $\mathscr{K}$-spaces are characterized exactly.

2. Quasi-Banach spaces. Throughout this paper all vector spaces will be real, although most arguments may be modified without difficulty to the complex case. 
A quasi-norm on a real vector space $X$ is a map ' $x \rightarrow\|x\|(X \rightarrow \boldsymbol{R})$ such that for some $K<\infty$,

$$
\begin{gathered}
\|x\|>0, \quad x \neq 0, \quad x \in X, \\
\|t x\|=|t|\|x\|, \quad t \in \boldsymbol{R}, \quad x \in X, \\
\|x+y\| \leqslant K(\|x\|+\|y\|), \quad x, y \in X .
\end{gathered}
$$

A quasi-norm induces a locally bounded topology on $X$ and conversely any locally bounded topology is given by a quasi-norm. A complete quasinormed space is called a quasi-Banach space. If in addition we have for some $0<p \leqslant 1$

(2.0.4)

$$
\|x+y\|^{p} \leqslant\|x\|^{p}+\|y\|^{p}, \quad x, y \in X,
$$

then $X$ is called a $p$-Banach space (or if $p=1$ a Banach space).

A quasi-Banach space $X$ is said to be $p$-convex for some $0<p \leqslant 1$ if there is a constant $A$ such that

$$
\left\|x_{1}+x_{2}+\ldots+x_{n}\right\| \leqslant A\left(\left\|x_{1}\right\|^{p}+\ldots+\left\|x_{n}\right\|^{p}\right)^{1 / p}
$$

for $x_{1}, \ldots, x_{n} \in X$. If $X$ is $p$-convex, it may be equivalently quasi-normed to be a $p$-Banach space. A theorem of Aoki and Rolewicz (see [17]) states that every quasi-Banach space is $p$-convex for some $p>0$. We shall repeatedly exploit this by assuming the quasi-norm on a given space satisfies $(2.0 .4)$ for some $p>0$.

We denote by $\left(\varepsilon_{n}: n \in \boldsymbol{N}\right)$ a sequence of independent random variables (or measurable functions) on $[0,1] \operatorname{such}$ that $\lambda\left(\varepsilon_{n}=+1\right)=\lambda\left(\varepsilon_{n}=-1\right)$ $=\frac{1}{8}$ where $\lambda$ is Lebesgue measure. We then say that a quasi-Banach space $X$ is type $p(0<p \leqslant 2)$ ([12], [13]) if for some constant $K<\infty$ we have

$$
\left(\int_{0}^{1}\left\|\sum_{i=1}^{n} \varepsilon_{i}(t) x_{i}\right\|^{p} d t\right)^{1 / p} \leqslant K\left(\sum_{i=1}^{n}\left\|x_{i}\right\|^{p}\right)^{1 / p} .
$$

If $X$ is $p$-convex, then $X$ is certainly type $p$.

We remark here that Kahane [4] shows that for a Banach space $X$ and $0<p<q<\infty$ there is a constant $K=K(p, q)$ such that

$$
\begin{aligned}
\left\{\int_{0}^{1}\left\|\sum \varepsilon_{i} x_{i}\right\|^{p} d t\right\}^{1 / p} & \leqslant\left\{\int_{0}^{1}\left\|\sum \varepsilon_{i} x_{i}\right\|^{\alpha} d t\right\}^{1 / \alpha} \\
& \leqslant K\left\{\int_{0}^{1}\left\|\sum \varepsilon_{i} x_{i}\right\|^{p} d t\right\}^{1 / p} .
\end{aligned}
$$

This means that we can change the exponent on the left of (2.0.6) without altering the definition.

In fact, (2.0.7) holds for quasi-Banach spaces; the modifications is Kahane's argument are minor but we include a proof for completeness.
THEOREM 2.1. Let $X$ be a quasi-Banach space. Then (2.0.7) holds. Proof. Let $\tilde{L}_{0}(X)$ be the space of $X$-valued simple functions on $[0,1]$ equipped with the topology of convergence in measure. Let $\operatorname{Rad}(\bar{X})$ be the subspace of functions of the form $\varepsilon_{1} x_{1}+\ldots+\varepsilon_{n} x_{n}$ for $x_{1}, \ldots, x_{n} \in \boldsymbol{X}$ and $n \in N$. We show that on $\operatorname{Rad}(X)$, the $L_{0}$-topology coincides with the stronger topology induced by any quasi-norm

$$
f \rightarrow\left\{\int_{0}^{1}\|f(t)\|^{p} d t\right\}^{1 / p} .
$$

We see this, we need only show that the set of $f \in \operatorname{Rad}(X)$ with $\lambda(\|f\| \geqslant 1)$

$<\frac{1}{8}$ is bounded in each $L_{p}$-norm.

Suppose $f=\varepsilon_{1} x_{1}+\ldots+\varepsilon_{n} x_{n}$ and

Let

$$
\lambda(\|f\|>r)=\alpha \text {. }
$$

$$
\begin{aligned}
& M(t)=\max _{1 \leqslant k \leqslant n}\left\|\sum_{i=1}^{k} \varepsilon_{i}(t) x_{i}\right\|, \quad 0 \leqslant t \leqslant 1, \\
& N(t)=\max _{1 \leqslant k \leqslant n}\left\|\sum_{i=k}^{n} \varepsilon_{i}(t) x_{i}\right\|, \quad 0 \leqslant t \leqslant 1 .
\end{aligned}
$$

Let $A_{k}(1 \leqslant k \leqslant n)$ be the set of $t$ such that

$$
\begin{aligned}
& \left\|\sum_{i=1}^{l} \varepsilon_{i}(t) x_{i}\right\|<K r, \quad 1 \leqslant l \leqslant k-1, \\
& \left\|\sum_{i=1}^{k} \varepsilon_{i}(t) x_{i}\right\| \geqslant K r
\end{aligned}
$$

(where $K$ is the modulus of concavity of the quasi-norm given by (2.0.3)).

Since $f$ has the same distribution as

and

$$
f^{*}=\varepsilon_{1} x_{1}+\ldots+\varepsilon_{k} x_{k}-\varepsilon_{k+1} x_{k+1}-\ldots-\varepsilon_{n} x_{n}
$$

$$
\begin{aligned}
\lambda\left(A_{k} \cap\left(\left\|f+f^{*}\right\| \geqslant 2 K r\right)\right) & \leqslant \lambda\left(A_{k} \cap(\|f\| \geqslant r)\right)+\lambda\left(A_{k} \cap\left(\left\|f^{*}\right\| \geqslant r\right)\right) \\
& =2 \lambda\left(A_{k} \cap(\|f\| \geqslant r)\right)
\end{aligned}
$$

and hence

$$
\lambda\left(A_{k}\right) \leqslant 2 \lambda\left(A_{k} \cap(\|f\| \geqslant r)\right),
$$

so that, summing over $k$,

$$
\lambda(M>K r) \leqslant 2 \alpha .
$$

Similarly, $\lambda(N>K r) \leqslant 2 \alpha$. 
Now if $t \in A_{k}$ and $\|f\| \geqslant 2 K^{2} r$,

$$
\left\|\sum_{i=1}^{k} \varepsilon_{i} x_{i}\right\| \geqslant \pi r
$$

and

$$
\left\|\sum_{i=1 k_{i}}^{n} \varepsilon_{i} x_{i}\right\| \geqslant K r \quad\left(\text { since }\left\|\sum_{i=1}^{k-1} \varepsilon_{i} x_{i}\right\|<K r\right)
$$

Hence

$$
\lambda\left(A_{k} \cap\left(\|f\| \geqslant 2 \dot{K^{2}} r\right)\right) \leqslant \lambda\left(A_{k} \cap\left(\left\|\sum_{i=k_{i}}^{n} \varepsilon_{i} x_{i}\right\| \geqslant K r\right)\right) \leqslant 2 \alpha \lambda\left(A_{k_{k}}\right)
$$

since these sets are independent. Summing over $k$,

$$
\lambda\left(\|f\| \geqslant 2 K^{2} r\right) \leqslant 4 \alpha^{2} .
$$

Thus if

$$
\begin{gathered}
\lambda(\|f\| \geqslant 1)<\frac{1}{8}, \\
\lambda\left(\|f\| \geqslant\left(2 K^{2}\right)^{n}\right)<4^{2^{n}-1}\left(\frac{1}{8}\right)^{2^{n}}<\left(\frac{1}{2}\right)^{2^{n}}
\end{gathered}
$$

and

$$
\int_{0}^{1}\|f\|^{p} d t<1+\sum_{n=0}^{\infty}\left(2 K^{2}\right)^{n p}\left(\frac{1}{2}\right)^{2^{n n}}=S_{p}<\infty .
$$

The galb $G(X)$ of a quasi-Banach space is the space of all sequences $\left\{a_{n}\right\}$ such that if $\left\|x_{n}\right\| \leqslant 1$, then $\left\{\sum_{k=1}^{n} a_{k} x_{k}\right\}$ is bounded. $G(X)$ is a quasiBanach space when quasi-normed by

$$
\left\|\left(a_{1}, a_{2}, \ldots\right)\right\|=\sup _{\left\|x_{k}\right\| \leqslant 1} \sup _{n}\left\|\sum_{k=1}^{n} a_{1} x_{k}\right\| .
$$

$X$ is said to be galbed by a space of sequences $E$ if, given $\left\|x_{n}\right\| \leqslant 1$, and $\left\{a_{n}\right\} \in E$, then $\sum_{k=1}^{n} a_{k_{0}} x_{z_{i}}$ is bounded (see Turpin [17] for a more detailed study of these notions).

An $F^{H}$-space is a complete metric topological vector space. A twisted. sum of two $F$-spaces $X$ and $Y$ is a space $Z$ which has a closed subspace $X_{0} \cong X$ such that $Z / X_{0} \cong X$. Thus there is a short exact sequence $0 \rightarrow X \rightarrow Z \rightarrow Y \rightarrow 0$. If every twisted sum of $X$ and $Y$ is a direct sum (i.e. $Z \cong X \oplus Y$ in the natural way) then we say that $(X, X)$ splits (the order is important here). If $(\boldsymbol{R}, X)$ splits, then $X$ is a $\mathscr{K}$-space ([8]).

If $X$ is a locally convex $\mathscr{K}$-space, then every twisted sum of $Y$ and $X$ with $Y$ locally convex is also locally convex ([6], Theorem 4.10) and this property characterizes locally convex $\mathscr{K}$-spaces.
3. Logconvex spaces and related classes. Let $\varphi$ denote the Orlicz function

$$
\varphi(t)= \begin{cases}t\left(1+\log \frac{1}{t}\right), & 0 \leqslant t \leqslant 1, \\ t, & t \geqslant 1\end{cases}
$$

(where $0 \log \infty=0 \log 0=0$ by convention). Then $l_{\varphi}$ is a locally bounded but non-locally convex Orlicz sequence space. The quasi-norm inducing the topology on $l_{\varphi}$ may be given by

$$
\|x\|_{\varphi}=\sup \left\{\xi: \sum_{i=1}^{\infty} \varphi\left(\xi^{-1}\left|x_{i}\right|\right) \leqslant 1\right\} .
$$

Our first result gives an equivalent quasi-norm.

THEOREM 3.1. An equivalent quasi-norm on $l_{\omega}$ is given by

$$
\|x\|_{p}^{*}=\|x\|_{1}+\sum_{i=1}^{\infty}\left|x_{i}\right| \log \frac{\|x\|_{1}}{\left|x_{i}\right|}
$$

where $\|x\|_{1}=\sum_{i=1}^{\infty}\left|x_{i}\right|$. that

Proof. Since $\|\cdot\|_{q}^{*}$ is easily seen to be homogeneous, it suffices to show

$$
0<\inf \left(\|x\|_{\varphi}^{*}:\|x\|_{\varphi}=1\right) \leqslant \sup \left(\|x\|_{\varphi}^{*}:\|x\|_{\varphi}=1\right)<\infty .
$$

If $\|x\|_{\varphi}=1$, then $\sum \varphi\left(\left|x_{i}\right|\right)=1$ and hence

$$
\sum\left|x_{i}\right|\left(1+\log \frac{1}{\left|x_{i}\right|}\right)=1 \text {. }
$$

Hence $\|x\|_{1} \leqslant 1$ and

$$
\|x\|_{\varphi}^{*} \leqslant\|x\|_{1}+\sum_{i=1}^{\infty}\left|x_{i}\right| \log \frac{1}{\left|x_{i}\right|}=\|x\|_{\varphi}=1 .
$$

Conversely,

$$
\|x\|_{p}^{*}=\|x\|_{p}-\|x\|_{1} \log \|x\|_{1}=1-\|x\|_{1} \log \|x\|_{1} \geqslant 1-\frac{1}{e}
$$

since $0 \leqslant\|t o\|_{1} \leqslant 1$.

Defrntmion 3.2. A quasi-Banach space $X$ is logeonvex if it is galbed by $l_{\varphi}$, i.e., whenever $x_{n} \in X$ and

$$
\sum \varphi\left(\left\|x_{n}\right\|\right)<\infty
$$


Exampua. The space $l_{\varphi}$ itself is logconvex; this follows easily from the fact that $p$ is submultiplicative at 0 (cf. Turpin [19], p. 79).

THeorem 3.3. A quasi-Banach space $X$ is logoonvex if and only if for some constant $O$ and any $x_{1}, \ldots, x_{n} \in X$

$$
\left\|x_{1}+x_{2}+\ldots+x_{n}\right\| \leqslant O\left[\sum_{i=1}^{n}\left\|x_{i}\right\|\left(1+\log \frac{S}{\left\|x_{i}\right\|}\right)\right]
$$

where $S=\sum_{i=1}^{n}\left\|x_{i}\right\|$.

Remark. (3.3.1) is oquivalent to

$$
\left\|x_{1}+\ldots+x_{n}\right\| \leqslant C\left(1+\sum_{i=1}^{n}\left\|x_{i}\right\| \log \frac{1}{\left\|x_{i}\right\|}\right)
$$

whenever $\left\|x_{1}\right\|+\ldots+\left\|x_{n}\right\| \leqslant 1$.

Proof. Let $I$ be an infinite set with $|I|=|X|$ and let $\left(x_{i}: i \in I\right)$ be the unit ball of $X$. If $X$ is logconvex the map $T: l_{\varphi}(I) \rightarrow X$ (where $l_{\varphi}(I)$ is the generalized sequence space of all $\left(\xi_{i}: i \in I\right)$ such that $\sum \varphi\left(\left|\xi_{i}\right|\right)<\infty$ defined by

$$
T(\xi)=\sum_{i \in I}^{\infty} \xi_{i} x_{i}
$$

is well-defined and continuous. Hence for some $C<\infty$

$$
\|T(\xi)\| \leqslant O\left(\|\xi\|_{1}+\sum_{i \in I}^{\infty}\left|\xi_{i}\right| \log \frac{\|\xi\|_{1}}{\left|\xi_{i}\right|}\right)
$$

and (3.3.1) follows easily.

Conversely, if (3.3.1) holds and

$$
\sum_{n=1}^{\infty} \varphi\left(\left\|x_{n}\right\|\right) \leqslant 1
$$

then

$$
\sum_{n=1}^{\infty}\left\|x_{n}\right\|\left(1+\log \frac{1}{\left\|x_{n}\right\|}\right) \leqslant 1
$$

and hence

$$
\left\|\sum_{n=k+1}^{l} x_{n}\right\| \leqslant C \sum_{n=k+1}^{l}\left\|x_{n}\right\|\left(1+\frac{1}{\log \left\|x_{n}\right\|}\right) \rightarrow 0 \text { as } \quad k, l \rightarrow \infty
$$

so that $\sum x_{n}$ converges. that

$L(1, \infty)$ denotes the space of measurable functions on $[0,1]$ such

$$
\|f\|=\sup x \lambda(|f|>x)<\infty
$$

Theorem 3.4. The space $L(1, \infty)$ is logconvex. [Added in proof: see [20].] Proof. Suppose $x_{1}, \ldots, x_{n} \in L(1, \infty)$ and let

$$
f=x_{1}+\ldots+x_{n} \text {. }
$$

Suppose also $\left\|x_{1}\right\|+\ldots+\left\|x_{n}\right\|=1$.

Fix $\tau, 0<\tau<1$ and let $A \subset(0,1)$ be a set of measure $\tau$. For each $i=1,2, \ldots, n$ let

$$
E_{i}=\left(\left|x_{i}\right|>2 \tau^{-1}\right)
$$

The $\lambda\left(E_{i}\right) \leqslant \frac{1}{2} \tau\left\|x_{i}\right\| ;$ hence if $E=E_{1} \cup \ldots \cup E_{n^{\prime}}$, then $\lambda(E) \leqslant \frac{1}{2} \tau$. Now

$$
\begin{aligned}
\inf _{t \in A}|f(t)| & \leqslant \inf _{t \in A \backslash E}|f(t)| \leqslant \frac{2}{\tau} \int_{A \backslash E}|f(t)| d t \\
& \leqslant \frac{2}{\tau} \sum_{i=1}^{n} \int_{A \backslash E}\left|x_{i}(t)\right| d t \leqslant \frac{2}{\tau} \sum_{i=1}^{n} \int_{A \backslash E_{i}}\left|x_{i}(t)\right| d t \\
& \leqslant \frac{2}{\tau} \sum_{i=1}^{n} \int_{\mathcal{A}} \min \left(\left|x_{i}(t)\right|, 2 \tau^{-1}\right) d t \leqslant \frac{2}{\tau} \sum_{i=0}^{n} \int_{0}^{\tau} \min \left(\frac{\left\|x_{i}\right\|}{u}, 2 \tau^{-1}\right) d u \\
& \leqslant \frac{2}{\tau} \sum_{i=1}^{n}\left(\left\|x_{i}\right\|+\int_{1 / 2 \tau\left\|x_{i}\right\|}^{\tau} \frac{\left\|x_{i}\right\|}{u} d u\right)=\frac{2}{\tau} \sum_{i=1}^{n}\left(\left\|x_{i}\right\|+\left\|x_{i}\right\| \log \frac{2}{\left\|x_{i}\right\|}\right) \\
& =\frac{1}{\tau}\left(2 \log 2+2+2 \sum_{i=1}^{n}\left\|x_{i}\right\| \log \frac{1}{\left\|x_{i}\right\|}\right) .
\end{aligned}
$$

Hence

$$
\|f\| \leqslant(2 \log 2+2)+2 \sum_{i=1}^{n}\left\|x_{i}\right\| \log \frac{1}{\left\|x_{i}\right\|}
$$

and so $L(1, \infty)$ is logconvex.

ExaMrple. Let $\left(g_{n}: n=1,2, \ldots\right)$ be a sequence of independent random variables each with the Cauchy distribution (i.e., with probability density function.

$$
f(x)=\frac{1}{\pi} \cdot \frac{1}{1+x^{2}}, \quad-\infty<x<\infty .
$$


Then $\left(\left|g_{n}\right|: n \in \boldsymbol{N}\right)$ is bounded in $L(1, \infty)$ and so if $a_{n} \geqslant 0, \sum a_{n}\left|g_{n}\right|$ converges in $L(1, \infty)$ if

$$
\sum_{n=1}^{\infty} a_{n}\left(1+\log \frac{1}{a_{n}}\right)<\infty
$$

and then

$$
\sum_{n=1}^{\infty} a_{n}\left|g_{n}(t)\right|<\infty \text { a.e. }
$$

L. Schwartz [18] shows that (3.4.1) is equivalent to (3.4.2). See Kahane [4] p. 97 for a similar example.

We now give another characterization of logconvex spaces; for this we require the following lemma.

Limaria 3.5. Suppose $\varepsilon>0$ and

$$
C_{\varepsilon}=\log \left[\sum_{k=1}^{\infty}\left(\frac{1}{k}\right)^{1+\varepsilon}\right](=\log \zeta(1+\varepsilon)) .
$$

- If $\xi_{1} \geqslant \xi_{2} \geqslant \ldots \geqslant \xi_{n} \geqslant 0$ and

then

$$
\xi_{1}+\xi_{2}+\ldots+\xi_{n}=1
$$

$$
\sum_{k=1}^{n} \xi_{k} \log k \leqslant \sum_{k=1}^{n} \xi_{k} \log \frac{1}{\xi_{k}} \leqslant(1+\varepsilon) \sum_{k=1}^{n} \xi_{k} \log k+C_{k} .
$$

Proof. Since $\xi_{k} \leqslant 1 / k$ the first inequality is clear. To prove the second, fix $n$ and let $C_{n}$ be the maximum of

$$
F\left(\xi_{1}, \xi_{2}, \ldots, \xi_{n}\right)=\sum_{k=1}^{n} \xi_{k} \log \frac{1}{\xi_{k}}-(1+\varepsilon) \sum_{k=1}^{n} \xi_{k} \log k
$$

subject to $\xi_{1} \geqslant \ldots \geqslant \xi_{n} \geqslant 0$ and $\xi_{1}+\ldots+\xi_{n}=1$. Then for some $\left(u_{1}, \ldots\right.$ $\left.\ldots, u_{n}\right), F\left(u_{1}, \ldots, u_{n}\right)=C_{n}$.

We claim first that $u_{1}>u_{2}>u_{3}>\ldots>u_{n}>0$. For if $l \leqslant n$ in the first index such that $u_{l}=0$ then a small increase in $u_{l}$ and decrease in $u_{l-1}$ increases $F$; a similar argument shows that $u_{i} \neq u_{j}$ if $i \neq j$. It follows that $\left(u_{1}, \ldots, u_{n}\right)$ is a local maximum of $F$ subject to the single condition $\xi_{1}+\ldots+\xi_{n}=1$. Hence there is a Lagrange multiplier $\lambda$ such that

i.e.,

$$
\frac{\partial F}{\partial \xi_{k}}\left(u_{1}, \ldots, u_{n}\right)=\lambda, \quad k=1,2, \ldots, n
$$

$$
\log \frac{1}{u_{k}}-(1+\varepsilon) \log k=\lambda+1
$$

Here $u_{k}=e^{-1-\lambda}\left(\frac{1}{k}\right)^{1+e}$ and so

$$
\left(\sum_{k=1}^{n}\left(\frac{1}{k}\right)^{1+\varepsilon}\right)=e^{\lambda+1}, \quad F\left(u_{1}, \ldots, u_{n}\right)=(\lambda-1)=C_{n},
$$

and hence $C_{n} \leqslant O_{e}$ and the result is proved.

THEOREM 3.6. A quasi-Banach space $X$ is logconvex if and only if for some $C<\infty$, whenever $x_{1}, \ldots, x_{n} \in X$

$$
\left\|x_{1}+\ldots+x_{n}\right\| \leqslant C \sum_{k=1}^{n}\left\|x_{k}\right\|(1+\log k)
$$

Proof. This follows immediately from the preceding lemma and Theorem 3.3.

Remark. This theorem essentially means that the Orlicz space $l_{p}$ is identical to the Lorentz space of all sequences $\left(a_{n}\right)$ such that $\sum a_{n}^{*}(1+$ $+\log n)<\infty$ where $\left(a_{n}^{*}\right)$ is decreasing re-arrangement of $\left(\left|a_{n}\right|\right)$. See [11] for similar results for convex Orlicz spaces and Lorentz spaces.

\section{Type in quasi-Banach spaces.}

THEOREM 4.1. Suppose $1<p \leqslant 2$; then a quasi-Banach space of type $p$ is convex.

Proof. Clearly if

$$
b_{n}=\sup _{\left\|x_{i}\right\| \leqslant 1} \inf _{\sigma_{i}= \pm 1}\left\|\sigma_{1} x_{1}+\sigma_{2} x_{2}+\ldots+\sigma_{n} x_{n}\right\|,
$$

then $b_{n}=o(n)$, and the result follows from Theorem 2.5 of [6].

THEOREM 4.2. Suppose $0<p<1$; then a quasi-Banach space $X$ of type $p$ is $p$-convex.

Pro of. We can and do suppose $X$ is an $r$-Banach space where $0<r$ $<p$. For each $n \in \boldsymbol{N}$, let $d_{n}$ be the least constant such that

$$
\left\|x_{1}+\ldots+x_{n}\right\| \leqslant d_{n}\left(\left\|x_{1}\right\|^{p}+\ldots+\left\|x_{n}\right\|^{p}\right)^{1 / p}
$$

for $x_{1}, \ldots, x_{n} \in X$. Suppose for any $n$

$$
\left\{\int_{0}^{1}\left\|\sum_{i=1}^{n} \varepsilon_{i}(t) x_{i}\right\|^{p} d t\right\}^{1 / p} \leqslant C\left(\sum_{i=1}^{n}\left\|x_{i}\right\|^{p}\right)^{1 / p}
$$

Then for any $x_{1}, \ldots, x_{n} \in X$ there exists $\sigma_{i}= \pm 1(1 \leqslant i \leqslant n)$ such that

$$
\left\|\sigma_{1} x_{1}+\ldots+\sigma_{n} x_{n}\right\| \leqslant C\left(\sum_{i=1}^{n}\left\|x_{i}\right\|^{p}\right)^{1 / p} .
$$



We may suppose that if $F=\left\{i: \sigma_{i}=-1\right\}$ then $\sum_{i \in F}\left\|x_{i}\right\|^{p} \leqslant \frac{1}{2} \sum_{i=1}^{n}\left\|x_{i}\right\|^{p}$.
Then

$$
\left\|\sum_{i \in F^{\prime}} x_{i}\right\| \leqslant 2^{-1 / p} d_{n}\left(\sum_{i=1}^{n}\left\|x_{i}\right\|^{p}\right)^{1 / p}
$$

and hence

$$
\left\|\sum_{i=1}^{n} x_{i}\right\|^{r} \leqslant\left\|\sum_{i=1}^{n} \sigma_{i} x_{i}\right\|^{r}+2^{r}\left\|\sum_{i \in H^{\prime}} x_{i}\right\|^{r} \leqslant\left(C^{r}+2^{r(1-1 / p)} d_{n}^{r}\right)\left(\sum_{i=1}^{n}\left\|x_{i}\right\|^{p}\right)^{r / p} .
$$

Thus

$$
d_{n}^{r} \leqslant U^{r}+2^{r(1-1 / p)} d_{n}^{r}
$$

so that

$$
d_{n} \leqslant \frac{C}{\left[1-\left(\frac{1}{2}\right)^{r(1 / 2)-1)}\right]^{1 / r}}
$$

As $\left\{a_{n}\right\}$ is bounded, $X$ is $p$-convex.

Remark. As is easily seen the hypothesis actually used in the proof is that $X$ satisfies

$$
\min _{\sigma_{i}= \pm 1}\left\|\sigma_{1} x_{1}+\ldots+\sigma_{n} x_{n}\right\| \leqslant C\left(\left\|x_{1}\right\|^{p}+\ldots+\left\|x_{n}\right\|^{p}\right)^{1 / p} .
$$

The same argument shows that if $b_{n}(X)=O\left(n^{1 / p}\right)(p<1)$ then $a_{n}(X)=O\left(n^{1 / p}\right)$ where

$$
\begin{aligned}
& b_{n}(X)=\sup _{\left\|x_{i}\right\| \leqslant 1} \min _{\sigma_{i}= \pm 1}\left\|\sigma_{1} x_{1}+\ldots+\sigma_{n} x_{n}\right\|, \\
& a_{n}(X)=\sup _{\left\|x_{i}\right\| \leqslant 1}\left\|x_{1}+\ldots+x_{n}\right\| .
\end{aligned}
$$

We do not know, however, if $a_{n}=O\left(n^{1 / p}\right)$ implies $X$ is $p$-convex when $p<1$.

When $p=1$ the above proot breaks down and we shall see later that a type 1 space need not be convex. It is tempting to conjecture that a type 1 space must at least be logconvex in view of the following theorem (the converse is clearly false-consider $l_{\varphi}$ ).

THEOREM 4.3. Let $X$ be a type 1 quasi-Banach space isomorphic to a subspace of $L_{0}$. Then $X$ is log convex.

Proof. By Nikisin's theorem ([7], [14]), $X$ embeds in $L(1, \infty)$. Now apply Theorem 3.5.

We have not been able to substantiate this conjecture and have only the following, whose proof we omit. It depends on rather more delicate handling of the argument in Theorem 4.2.
THookm 4.4. Suppose $X$ is a type 1 quasi-Banach space; then for some $C<\infty$ and any $x_{1}, \ldots, x_{n} \in X$

$$
\left\|x_{1}+\ldots+x_{n}\right\| \leqslant C(1+\log n)\left(\left\|x_{1}\right\|+\ldots+\left\|x_{n}\right\|\right) .
$$

In view of the above results we shall only consider type when $1 \leqslant p \leqslant 2$.

5. Twisted sums. Suppose $X$ and $Y$ are quasi-Banach spaces and $\boldsymbol{Z}$ is a twisted sum of $Y$ and $X$, so that $Z$ has a subspace isomorphic to $\boldsymbol{Y}$ such that $Z / Y \cong X$. Then (cf. [6], [8]) there is a map $I F: X \rightarrow Y$ satisfying

$$
F(t x)=t F^{\prime}(x), \quad t \in \boldsymbol{R}, x \in \mathcal{X},
$$

(5.0.2) $\quad\left\|F\left(x_{1}+x_{2}\right)-F\left(x_{1}\right)-F\left(x_{2}\right)\right\| \leqslant K\left(\left\|x_{1}\right\|+\left\|x_{2}\right\|\right), \quad x_{1}, x_{2} \in \boldsymbol{X}$,

where $K$ is independent of $x_{1}, x_{2}$, such that $Z$ is isomorphic to the space $Y \oplus_{F} X$, i.e., the Cartesian sum $Y \oplus X$ quasinormed by

$$
\|(y, x)\|=\|y-F(x)\|+\|x\| \text {. }
$$

Conversely, given any such quasilinear map $F$ satisfying (5.0.1) and (5.0.2), then $Y \oplus_{F} X$ is a twisted sum of $Y$ and $X$.

Suppose then $F: X \rightarrow Y$ is a fixed quasilinear map. We define for a finite subset $\left\{x_{1}, \ldots, x_{n}\right\}$ of $X$

$$
A\left(x_{1}, \ldots, x_{n}\right)=F\left(x_{1}+\ldots+x_{n}\right)-\sum_{i=1}^{n} F\left(x_{i}\right) .
$$

We now state the properties of $\triangle$.

LEMMA 5.1. (1) If $A_{1}, A_{2}, \ldots, A_{m}$ are disjoint subsets of $\{1,2, \ldots, n\}$ such that $A_{1} \cup \ldots \cup A_{m}=\{1,2, \ldots, n\}$ and

$$
u_{i}=\sum_{j \in A_{i}} x_{j},
$$

shen

$$
\Delta\left(x_{1}, \ldots, x_{n}\right)=\Delta\left(u_{1}, u_{2}, \ldots, u_{m}\right)+\sum_{i=1}^{m} \Delta\left(x_{j}: j \in A_{i}\right) .
$$

(2) $\Delta(x)=0$.

(3) $\left\|\Delta\left(x_{1}, \infty_{2}\right)\right\| \leqslant K\left(\left\|x_{1}\right\|+\left\|x_{2}\right\|\right)$.

(4) Fror some $s, 0<s \leqslant 1$ and $M<\infty$

$$
\left\|\Delta\left(x_{1}, \ldots, x_{n}\right)\right\| \leqslant M\left(\left\|x_{1}\right\|^{s}+\ldots+\left\|x_{n}\right\|^{s}\right)^{1 / s}
$$

for $x_{1}, \ldots, x_{n} \in X$.

Proof. (1)-(3) are obvious and (4) is shown in [6].

Now suppose that $W$ is any quasi-Banach space. We define $d_{n}=d_{n}(W)$ to be a least constant such that

$$
\left\|w_{1}+\ldots+w_{n}\right\| \leqslant d_{n}\left(\left\|w_{1}\right\|+\ldots+\left\|w_{n}\right\|\right)
$$


whenever $w_{1}, \ldots, w_{n} \in W$. We also define $\delta_{n}=\delta_{n}(W)$ to be the least constant such that

$$
\left\{\int_{0}^{1}\left\|\sum_{i=1}^{n} \varepsilon_{i} w_{i}\right\|^{2} d t\right\}^{1 / 2} \leqslant \delta_{n}\left(\sum_{i=1}^{n}\left\|w_{i}\right\|^{2}\right)^{1 / 2}
$$

The sequence $\left\{\delta_{n}\right\}$ has been studied for Banach space in [1], [2] and [13]. It is easy enough to see that both sequences $\left\{d_{n}\right\}$ and $\left\{\delta_{n}\right\}$ aro submultiplicative $\left(d_{m n} \leqslant d_{m} d_{n}, \delta_{m n} \leqslant \delta_{m} \delta_{n}\right)$.

For a quasilinear maip $F: X \rightarrow Y$ we define $\theta_{n}=o_{n}\left(F^{\prime}\right)$ to be the loast constant such that

$$
\left\|\Delta\left(x_{1}, \ldots, x_{n}\right)\right\| \leqslant c_{n}\left(\left\|x_{1}\right\|+\ldots+\left\|x_{n}\right\|\right), \quad x_{1}, \ldots, x_{n} \in X,
$$

and $\gamma_{n}=\gamma_{n}(F)$ to be the least constant such that

$\left\{\int_{0}^{1}\left\|\Delta\left(\varepsilon_{1} x_{1}, \varepsilon_{2} x_{2}, \ldots, \varepsilon_{n} x_{n}\right)\right\|^{2} d t\right\}^{1 / 2} \leqslant \gamma_{n}\left(\left\|x_{1}\right\|^{2}+\ldots+\left\|x_{n}\right\|^{2}\right)^{1 / 2}, \quad x_{1}, \ldots, x_{n} \in X$.

Our first result is simply a generalization of a result of Inflo, Lindenstrauss and Pisier.

THEOREM 5.2. Suppose $0<r \leqslant 1$ and $Y$ is an $r$-Banach space. Iror $m, \dot{n} \in \mathbf{N}$

$$
\begin{gathered}
c_{m n}^{r} \leqslant c_{m}^{r} \partial_{n}^{r}(X)+c_{n}^{r} \partial_{m}^{r}(Y), \\
\gamma_{m n}^{r} \leqslant \gamma_{m}^{r} \delta_{n}^{r}(X)+\gamma_{n}^{r} \delta_{m}^{r}(Y) .
\end{gathered}
$$

Proof. (5.2.1). Suppose $x_{1}, x_{2}, \ldots, x_{m n} \in X$. Let

$$
u_{i}=\sum_{(i-1) n+1}^{i n} x_{j}, \quad i=1,2, \ldots, m .
$$

Then $\left\|\Delta\left(u_{1}, \ldots, u_{m}\right)\right\| \leqslant c_{m} \sum_{i=1}^{m}\left\|u_{i}\right\| \leqslant c_{m} d_{n}(X) \sum_{i=1}^{m n}\left\|x_{i}\right\|$ and

$$
\begin{aligned}
\left\|\sum_{i=1}^{m} \Delta\left(x_{j}:(i-1) n<j \leqslant i n\right)\right\| & \leqslant d_{m}(Y) \sum_{i=1}^{m}\left\|\Delta\left(x_{j}:(i-1) n<j \leqslant i n\right)\right\| \\
& \leqslant d_{m}(Y) c_{n} \sum_{i=1}^{m n}\left\|x_{i}\right\|
\end{aligned}
$$

and (5.2.1) follows from Leinma 5.1.

(5.2.2). Let

$$
u_{i}(t)=\sum_{(i-1) n+1}^{i n} \varepsilon_{j}(t) x_{j}
$$

Then

$\int_{0}^{1}\left\|\Delta\left(u_{1}(t), u_{2}(t), \ldots, u_{m}(t)\right)\right\|^{2} d t=\int_{0}^{1} \int_{0}^{1}\left\|\Delta\left(\varepsilon_{1}(s) u_{1}(t), \ldots, \varepsilon_{m}(s) u_{m}(t)\right)\right\|^{2} d s d t$

(by sylumotry)

$$
\leqslant \int_{0}^{1} \gamma_{m}^{2} \sum_{i=1}^{m}\left\|u_{i}(t)\right\|^{2} d t \leqslant \gamma_{m}^{2} \delta_{n}^{2}(X) \sum_{i=1}^{m n}\left\|x_{i}\right\|^{2}
$$

Also, by a similar atrgument,

$$
\int_{0}^{1}\left\|\sum_{i=1}^{m} \Lambda\left(s_{j} ; x_{j}:(i,-1) n<j \leqslant i n\right)\right\|^{2} d t \leqslant \delta_{m}^{2}(Y) \gamma_{n}^{2} \sum_{i=1}^{m n}\left\|x_{i}\right\|^{2}
$$

and (5.2.2) now follows firom Lemmat 5.1 and the convexity of the $L_{2 / r}$-norm.

Lemma 5.3. If $p>0$, there exists $\alpha=\alpha(p) \geqslant 0$ and $O=C(p)$ such that for $x_{1}, \ldots, x_{n} \in X$

$$
\left\|\Delta\left(x_{1}, \ldots, x_{n}\right)\right\| \leqslant C\left(\sum_{k=1}^{n} k^{a}\left\|x_{k}\right\|^{p}\right)^{1 / p}
$$

Proof. By Lemma 5.1 we can find $s>0$ and $M<\infty$ such that

$$
\left\|A\left(x_{1}, \ldots, x_{n}\right)\right\| \leqslant M\left(\sum_{k=1}^{n}\left\|x_{\eta_{i}}\right\|^{s}\right)^{1 / s} .
$$

Thus for $0<p \leqslant s, \alpha=0$ will suffice. Now suppose $s<p<\infty$, and choose $\theta>$. 1. Let $e=\left(\sum_{k=1}^{\infty} k^{-\theta}\right)^{-1}$. Then $\sum_{k=1}^{\infty} c k^{-\theta}=1$ and hence

$$
\left(\sum_{k=1}^{n}\left\|x_{l s}\right\|^{s}\right)^{1 / s}=\left(\sum_{k=1}^{n} c k^{-0}\left\|x_{l c}\right\|^{s} c^{-1} k_{k}^{0}\right)^{1 / s} \leqslant\left(\sum_{k=1}^{n} c k^{-0}\left\|x_{k}\right\|^{p} o^{-p / s} k_{k}^{\theta p / s}\right)^{1 / p}
$$

and hence $\alpha=\theta(p / s-1)$ will suffice.

\section{Twisted sums with unequal convexity.}

LWMMA 6.1. Suppose $\mu>v \geq 0$, and $X$ is an $r$ Banach space.

(6.1.1) If $d_{n}(Y)=O\left(n^{\mu^{n}}\right)$ and $a_{n}(X)=O\left(n^{\prime \prime}\right)$, then $o_{n} \cdots O\left(n^{\mu}\right)$.

(6.1.2) If $\delta_{n}(X)=O\left(n^{\mu}\right)$ and $\delta_{n}(X)=O\left(n_{j}^{\prime \prime}\right)$, then $\gamma_{n}=O\left(n^{\mu}\right)$.

Remark. The roles of $X$ and $Y$ may be interchanged in this lemma.

Proof. Suppose $d_{n}(Y) \leqslant a n^{\prime \prime}$ and $d_{n}(X) \leqslant b n^{\prime}$. Solect $N$ so that $b N^{\nu-\mu}<$ 1. Lot $\theta_{k}=\left(o_{N^{k}} N^{-k^{k}}\right)^{r}$. Then .

$$
a_{N^{k}}^{r}: a_{N^{k-1}}^{r} d_{N}^{r}(X)+e_{N}^{r} d_{N^{k-1}}^{r}(Y)
$$


so that

$$
\theta_{k} \leqslant\left(b N^{v-\mu}\right)^{r} \theta_{k-1}+a \theta_{1}
$$

and hence $\left\{\theta_{k}\right\}$ is bounded. Hence $e_{n}=O\left(n^{\mu}\right)$.

(6.1.2) has a similar proof.

THEOREM 6.2. Suppose that $0<p, q \leqslant 1$ and $p \neq q$, and that $X$ is a p-convex quasi-Banach space and $Y$ is a q-convex quasi-Banach space. Then any twisted sum $Y \oplus_{F} X$ of $Y$ and $X$ is $\min (p, q)$-convex.

Proof. The case $q>p$ is proved in [6]. We therefore assume that $q<p$ and that $Y$ is a $q$-Banach space and $X$ is a $p$-Banach space. Then

$$
d_{n}(Y) \leqslant n^{1 / \alpha-1}, \quad d_{n}(X) \leqslant n^{1 / p-1},
$$

and hence for any quasilinear map $F: X \rightarrow Y$

for some $C$.

$$
c_{n}(F) \leqslant C n^{1 / q-1},
$$

Now suppose $x_{1}, \ldots, x_{n} \in X$ is non-zero and

$$
\left\|x_{1}\right\|^{\alpha}+\ldots+\left\|x_{n}\right\|^{a}=1 \text {. }
$$

Let $A_{m}=\left\{i: 2^{-m}<\left\|x_{i}\right\| \leqslant 2 \cdot 2^{-m}\right\}, m=1,2,3, \ldots$ Then for some $N$, $A_{1}, \ldots, A_{N}$ partitions $\{1,2, \ldots, n\}$. Let

$$
u_{m}=\sum_{i \in A_{m}} x_{i} .
$$

Then, if we make the convention $\Delta(\varnothing)=0$ and $\sum_{\varnothing} x_{i}=0$,

$$
\left\|\Delta\left(x_{1}, \ldots, x_{n}\right)\right\|^{q} \leqslant\left\|\Delta\left(u_{1}, \ldots, u_{N}\right)\right\|^{q}+\sum_{i=1}^{N}\left\|\Delta\left(x_{j}: j \in A_{i}\right)\right\|^{q} .
$$

Now

$$
\left\|\Delta\left(x_{j}: j \in A_{i}\right)\right\| \leqslant C\left|A_{i}\right|^{1 / \alpha-1} \sum_{j \in A_{i}}\left\|x_{j}\right\| \leqslant 2 C\left|A_{i}\right|^{1 / \alpha} 2^{-i},
$$

so that

$$
\left\|\Delta\left(x_{j}: j \in A_{i}\right)\right\|^{q} \leqslant(2 C)^{q}\left|A_{i}\right| 2^{-i q} \leqslant(2 C)^{q} \sum_{j \in A_{i}}\left\|x_{j}\right\|^{q} .
$$

Hence

$$
\sum_{i=1}^{N}\left\|\Delta\left(x_{j}: j \in A_{i}\right)\right\|^{q} \leqslant(2 C)^{q} \sum_{i=1}^{n}\left\|x_{i}\right\|^{q}=(2 C)^{q} .
$$

Now by Lemma 5.3 there is a constant $M$ and $\alpha \geqslant 0$ so that

$$
\left\|\Delta\left(w_{1}, \ldots, w_{l}\right)\right\|^{p} \leqslant M \sum_{k=1}^{l} k^{a}\left\|w_{k}\right\|^{p}, \quad w_{1}, \ldots, w_{l} \in X .
$$

Henco

$$
\begin{aligned}
\left\|\Delta\left(u_{1}, \ldots, u_{N}\right)\right\|^{p} & \leqslant M \sum_{k=1}^{N} k^{\alpha}\left\|u_{k_{k}}\right\|^{p} \leqslant M \sum_{k=1}^{N} k^{\alpha} \sum_{i \in A_{k}}\left\|x_{i}\right\|^{p} \\
& \leqslant M^{*} \sum_{i=1}^{n}\left\|x_{i}\right\|^{p}\left(\log \frac{2}{\left\|x_{i}\right\|}\right)^{a}
\end{aligned}
$$

where $M^{*}=M / \log 2$.

Now $\sup \xi^{y-q}(\log (2 / \xi))^{a}=0<\infty$ and hence if $M^{* *}=\theta M^{*}$

$$
\left\|\Delta\left(u_{1}, \ldots, u_{N}\right)\right\|^{p} \leqslant M^{* *} \sum_{i=1}^{n}\left\|o_{i}\right\|^{\alpha}=M^{* *} .
$$

Hence

$$
\left\|\Delta\left(x_{1}, \ldots, x_{n}\right)\right\|^{\alpha} \leqslant\left(M^{* *}\right)^{q}+(2 C)^{\alpha}
$$

where both $M^{* * *}$ and $C$ are independent of $x_{1}, \ldots, x_{n}$. We conclude that for any $x_{1}, \ldots, x_{n}$

$$
\left\|\Delta\left(x_{1}, \ldots, x_{n}\right)\right\|^{q} \leqslant D \sum_{i=1}^{n}\left\|x_{i}\right\|^{q} .
$$

Now suppose $\left(y_{i}, x_{i}\right) \in Y \oplus_{F} X$. Then

$$
\begin{aligned}
\left\|\left(\sum y_{i}, \sum x_{i}\right)\right\|^{\alpha} & =\left(\left\|\sum y_{i}-F\left(\sum x_{i}\right)\right\|+\left\|\sum x_{i}\right\|\right)^{\alpha} \\
& \leqslant\left(\left\|\sum y_{i}-F^{\prime}\left(\sum x_{i}\right)\right\|^{\alpha}+\left\|\sum x_{i}\right\|^{\alpha}\right) \\
& \leqslant\left(\left\|\sum\left(y_{i}-F^{\prime}\left(x_{i}\right)\right)\right\|^{\alpha}+\left\|\Delta\left(x_{1}, \ldots, x_{n}\right)\right\|^{\alpha}+\left\|\sum x_{i}\right\|^{\alpha}\right) \\
& \leqslant 2 D\left(\sum\left\|x_{i}\right\|^{\alpha}+\sum\left\|y_{i}-F^{\prime}\left(x_{i}\right)\right\|^{\alpha}\right) \\
& \leqslant 2^{1 / \alpha-1} D \sum\left\|\left(x_{i}, y_{i}\right)\right\|^{\alpha}
\end{aligned}
$$

and so $Y \oplus_{H} X$ is $q$-convex.

THxorfic 6.4. Suppose that $X$ is a quasi-Banaeh space of type $p \quad(1 \leqslant p$ $\leqslant 2)$ and $Y$ is a quasi-Banash space of type $q(1 \leqslant q \leqslant 2)$. Then if $q<p$, any twisted sum $Y \oplus_{1,} X$ is of typo $q$.

Proof. This proof mimics Theorem 6.2 . Wo assume that $Y$ is an $r$-Banach space where $r \leqslant 1$; of course, if $q>1$, we may take $r=1$. We suppose that if $x_{1}, \ldots, x_{n} \in X$ and $y_{1}, \ldots, y_{n} \in Y$, then

$$
\begin{aligned}
& \left\{\int_{0}^{1}\left\|\sum_{i=1}^{n} \varepsilon_{i}(t) x_{i}\right\|^{p} d t\right\}^{1 / \alpha} \leqslant o\left(\sum\left\|x_{i}\right\|^{p}\right)^{1 / 2 x}, \\
& \left\{\int_{0}^{1}\left\|\sum_{i=1}^{n} \varepsilon_{i}(t) y_{i}\right\|^{\alpha} d t\right\}^{1 / \alpha} \leqslant o\left(\sum\left\|x_{i}\right\|^{\alpha}\right)^{1 / \alpha},
\end{aligned}
$$


and that

$$
\left\|\Delta\left(x_{1}, \ldots, x_{n}\right)\right\| \leqslant M\left(\sum_{k=1}^{n} l_{k}^{a}\left\|x_{k}\right\|^{p}\right)^{1 / p}
$$

as in Lemma 5.3.

We have $\delta_{n}(\bar{Y})=O\left(n^{1 / q-1 / 2}\right)$ and $\delta_{n}(X)=O\left(n^{1 / p-1 / 2}\right)$ and hence

$$
\gamma_{n}\left(F^{\prime}\right) \leqslant C n^{1 / q-1 / 2}, \quad n \in \boldsymbol{N} .
$$

Suppose $x_{1}, \ldots, x_{n} \in X$ are non-zero and

$$
\left\|x_{1}\right\|^{q}+\ldots+\left\|x_{n}\right\|^{q}=1 .
$$

Let $A_{m}=\left\{i: 2^{-m}<\left\|x_{i}\right\| \leqslant 2 \cdot 2^{-m}\right\}$ and suppose $A_{1}, \ldots, A_{N}$ positions $\{1,2, \ldots, N\}$. As before we make the convention $\Delta(\varnothing)=0$ and $\sum_{i \in \Phi} x_{i}=0$.
Then if

$$
\begin{aligned}
u_{i}(t) & =\sum_{j \in A_{i}} \varepsilon_{j}(t) x_{i}, \quad 0 \leqslant t \leqslant 1 \\
\Delta\left(\varepsilon_{1}(t) x_{1}, \ldots, \varepsilon_{n}(t) x_{n}\right) & =\Delta\left(u_{1}(t), \ldots, u_{N}(t)\right)+\sum_{i=1}^{N} \Delta\left(\varepsilon_{j}(t) x_{j}: j \in A_{i}\right) .
\end{aligned}
$$

Now by symmetry

$$
\begin{aligned}
& \int_{0}^{1}\left\|\sum_{i=1}^{N} \Delta\left(\varepsilon_{j}(t) x_{j}: j \in A_{i}\right)\right\| \alpha d t=\int_{0}^{1} \int_{0}^{1}\left\|\sum_{i=1}^{N} \varepsilon_{j}(s) \Delta\left(\varepsilon_{j}(t) x_{j}: \quad j \in A_{i}\right)\right\|^{\alpha} d s d t \\
& \leqslant e^{\alpha} \int_{0}^{1} \sum_{i=1}^{N}\left\|\Delta\left(\varepsilon_{j}(t) x_{j}: j \in A_{i}\right)\right\|^{\alpha} d t \\
& \text {. } \quad \leqslant c^{q} \sum_{i=1}^{N}\left\{\int_{0}^{1}\left\|\Delta\left(\varepsilon_{j}(t) x_{j}: j \in A_{i}\right)\right\|^{2} d t\right\}^{q / 2} \\
& \leqslant c^{q} \sum_{i=1}^{N} C^{q}\left|A_{i}\right|^{1-q / 2}\left(\sum_{j \in A_{i}}\left\|x_{j}\right\|^{2}\right)^{q / 2} \\
& \leqslant 2^{q / 2} a^{q} C^{q} \sum_{i=1}^{N}\left|A_{i}\right| 2^{-i q} \leqslant 2^{q / 2} e^{q} C^{q} \sum_{i=1}^{n}\left\|\alpha_{i}\right\|^{q} \leqslant 2^{\alpha / 2} o^{q} O^{q q} .
\end{aligned}
$$

Also

$$
\begin{aligned}
\int_{0}^{1}\left\|\Delta\left(u_{1}(t), \ldots, u_{N}(t)\right)\right\|^{p} d t & \leqslant M^{p} \cdot \int_{0}^{1} \sum_{k=1}^{N} l c^{a}\left\|u_{k}(t)\right\|^{p} d t \leqslant M^{p} e^{p} \sum_{k=1}^{N} k^{a} \sum_{j \in A_{k}}^{\top}\left\|x_{j}\right\|^{p} \\
& \leqslant M^{*} \sum_{i=1}^{n}\left\|x_{j}\right\|^{p}\left(\log \frac{2}{\left\|x_{j}\right\|}\right)^{a} \leqslant M^{* *}
\end{aligned}
$$

as in Theorem 6.2, whare $M^{* * 4}$ is independent of $x_{1}, \ldots, x_{n}$. Hence

$$
\begin{gathered}
\left\{\int_{0}^{1}\left\|\Delta\left(\varepsilon_{1} x_{1}, \ldots, \varepsilon_{n} x_{n}\right)\right\|^{q} d t\right\}^{r / q} \leqslant\left\{\int_{0}^{1}\left\|\Delta\left(u_{1}(t), \ldots, u_{N}(t)\right)\right\|^{q} d t\right\}^{r / q}+ \\
+\left\{\iint_{0}^{1}\left\|\sum_{i=1}^{N} \Delta\left(\varepsilon_{j} x_{j}: j \in A_{i}\right)\right\|^{\alpha} d t\right\}^{r / \alpha} \\
\leqslant\left(M M^{* * *}\right)^{r}+2^{r / 2} i^{r} C^{r} .
\end{gathered}
$$

It follows asusily that for any $x_{1}, \ldots, x_{n}$

$$
\left\{\int_{0}^{1}\left\|A\left(\varepsilon_{1} x_{1}, \ldots, \varepsilon_{n} x_{n}\right)\right\|^{\mid t} d t\right\}^{1 / 1} \leqslant D\left\{\sum\left\|x_{i}\right\|^{q}\right\}^{1 / q} .
$$

Now suppose $\left(y_{i}, x_{i}\right) \subset Y \mathscr{O}_{1} X(1 \leq i \leq n)$. Then

$$
\begin{aligned}
& \left\{\int_{0}^{1}\left\|\sum_{i=1}^{n} s_{i}(t)\left(y_{i}, x_{i}\right)\right\|^{\alpha} d t\right\}^{1 / t} \\
& =\left\{\int_{0}^{1}\left(\left\|\sum \varepsilon_{i}(t) y_{i} \cdots \cdots\left(\sum \varepsilon_{i}(t) x_{i}\right)\right\|+\left\|\sum \varepsilon_{i}(t) x_{i}\right\|\right)^{\alpha} d t\right\}^{1 / \alpha} \\
& \left\{\int_{0}^{1}\left\|\sum \varepsilon_{i}(t) y_{i}-H^{\prime}\left(\sum \varepsilon_{i}(t) x_{i}\right)\right\|^{q} d t\right\}^{1 / q}+o\left(\sum\left\|x_{i}\right\|^{q}\right)^{1 / q}, \\
& \left\|\sum \varepsilon_{i}(t) y_{i}-W^{\prime}\left(\sum \varepsilon_{i}(t) x_{i}\right)\right\| \leqslant 2^{1 / n-1}\left(\left\|\sum \varepsilon_{i}(t)\left(y_{i}-F^{\prime}\left(x_{i}\right)\right)\right\|+\left\|A\left(x_{1}, \ldots, x_{n}\right)\right\|\right)
\end{aligned}
$$

so

$$
\begin{aligned}
& \left\{\int_{0}^{1}\left\|\sum \varepsilon_{i}(t)\left(y_{i}, x_{i}\right)\right\|^{a} d t\right\}^{1 / \alpha} \\
& \left.\leqslant 2^{1 / r-1} c\left(\sum\right\urcorner\left\|y-F^{\prime}\left(x_{i}\right)\right\|^{q}\right)^{1 / q}+\left(2^{1 / r-1} D+c\right)\left(\sum\left\|x_{i}\right\|^{\|^{2}}\right)^{1 / q} \\
& \leqslant\left(2^{1 / r-1}(\theta+D)+\theta\right)\left(\sum\left(\left\|x_{i}\right\|+\left\|y-W^{\prime}\left(x_{i}\right)\right\|^{\alpha}\right)^{\alpha}\right)^{1 / \alpha} \\
& \leftarrow K\left(\sum\left\|\left(y_{i}, m_{i}\right)\right\|^{q}\right)^{1 / \alpha} \text {, }
\end{aligned}
$$

i.e., $Y \oplus_{m} X$ is tiypo $q$.

Wo now show that a tiype 1 space need not bo convex.

In $[6],[15]$ and $[16]$ it is shown that one can construct a non-eonvex twisted sum of $\boldsymbol{R}$ and an Banach space. This is type 1 by the next theorem.

THжонті 6.5. Suppose $X$ is a type $p$ quasi-Banach space and $X$ is a type $q$ Banach space where $q>p$. Then any twisted sum $Y \oplus_{F} X$ is type $p$.

Proof. Here our techniques are rather different. We use a result of Kahane [4] that there is a constant $K=K(p, q)$ such that for any 
elements $u_{1}, \ldots, u_{n}$ of $X$

$$
\left\{\int_{0}^{1}\left\|\sum \varepsilon_{i} u_{i}\right\|^{\alpha} d t\right\}^{1 / \alpha} \leqslant K\left\{\int_{0}^{1}\left\|\sum \varepsilon_{i} u_{i}\right\|^{p} d t\right\}^{1 / p} .
$$

[For the case $p=1$, we apply Theorem 2.1.]

Suppose $e$ has the same meaning as in Theorem 6.4. Let $\theta_{n}$ be the least constant such that

$$
\left\{\int\left\|\Delta\left(\varepsilon_{1} x_{1}, \ldots, \varepsilon_{n} x_{n}\right)\right\|^{\alpha} d t\right\}^{1 / q} \leqslant \theta_{n}\left\{\sum_{i=1}^{n}\left\|x_{i}\right\|^{p}\right\}^{1 / p} .
$$

Suppose $\left\|x_{1}\right\|^{p}+\ldots+\left\|x_{n}\right\|^{p}=1$ and $\left\|x_{n}\right\|^{p} \geqslant 1 / N$. Then

$$
\Delta\left(\varepsilon_{1} x_{1}, \ldots, \varepsilon_{n} x_{n}\right)=\Delta\left(u(t), \varepsilon_{n}(t) x_{n}\right)+\Delta\left(\varepsilon_{1} x_{1}, \ldots, \varepsilon_{n-1} x_{n-1}\right)
$$

where $u(t)=\sum_{i=1}^{n-1} \varepsilon_{i}(t) x_{i}$. Now

$$
\left\{\int_{0}^{1}\left\|\Delta\left(u(t), \varepsilon_{n}(t) x_{n}\right)\right\| \|^{q} d t\right\}^{p / q} \leqslant \theta_{2}^{\nu}\left\{\int_{0}^{1}\left(\|u(t)\|\left\|^{p}+\right\| x_{n} \|^{p}\right)^{q / p} d t\right\}^{p / q}
$$

by the usual symmetrization argument.

Hence

$$
\begin{aligned}
\left\{\int_{0}^{1}\left\|\Delta\left(u(t), \varepsilon_{n}(t) x_{n}\right)\right\| \|^{q} d t\right\}^{p / q} & \leqslant \theta_{2}^{p}\left[\left(\int_{0}^{1}\|u(t)\|^{q} d t\right)^{p / q}+\left\|x_{n}\right\|^{p}\right] \\
& \leqslant \theta_{2}^{p}\left[K^{p} c^{p} \sum_{i=1}^{n-1}\left\|x_{i}\right\|^{p}+\left\|x_{n}\right\|^{p}\right] \leqslant K^{p} \theta_{2}^{p} c^{p} .
\end{aligned}
$$

Hence if $\left\|x_{1}\right\|^{p}+\ldots+\left\|x_{n}\right\|^{p}=1$ and $\max \left\|x_{i}\right\|^{p} \geqslant N^{-1}$,

$$
\left\{\int_{0}^{1}\left\|\Delta\left(\varepsilon_{1} x_{1}, \ldots, \varepsilon_{n} x_{n}\right)\right\|^{\alpha} d t\right\}^{1 / q} \leqslant \theta_{n-1}(1-1 / N)^{1 / p}+K \theta_{2} c .
$$

Now suppose $\left\|x_{1}\right\|^{p}+\ldots+\left\|x_{n}\right\|^{p}=1$ and $\max \left\|x_{i}\right\|^{p}<N^{-1}$. Then it is possible to subdivide $\{1,2, \ldots, n\}$ into $N$ sets $A_{1}, \ldots, A_{N}$ such that

Then let

$$
\sum_{i \in A_{j}}\left\|x_{i}\right\|^{p} \leqslant 2 / N, \quad j=1,2, \ldots, N
$$

$$
\begin{gathered}
u_{j}(t)=\sum_{i \in A_{j}} \varepsilon_{i}(t) x_{i} \\
\Delta\left(\varepsilon_{1} x_{1}, \ldots, \varepsilon_{n} x_{n}\right)=\Delta\left(u_{1}(t), \ldots, u_{N}(t)\right)+\sum_{i=1}^{N} \Delta\left(\varepsilon_{i} x_{i}: i \in A_{j}\right)
\end{gathered}
$$

and by symmetrization

$$
\begin{aligned}
\left\{\int_{0}^{1}\left\|\Delta\left(u_{1}(t), \ldots, u_{N}(t)\right)\right\|^{q} d t\right\}^{p / q} & \leqslant \theta_{N}^{p}\left\{\int_{0}^{1}\left(\sum_{i=1}^{N}\left\|u_{i}(t)\right\|^{p}\right)^{\alpha / p} d t\right\}^{p / \alpha} \\
& \leqslant \theta_{N}^{p} \sum_{i=1}^{N}\left\{\int_{0}^{1}\left\|u_{i}(t)\right\|^{\alpha} d t\right\}^{p / q} \\
& \leqslant \theta_{N}^{p} \sum_{i=1}^{N} K^{p} o^{p} \sum_{i \in A_{j}}\left\|x_{i}\right\|^{p} \leqslant \theta_{N}^{p} H^{p} o^{p}, \\
\left\{\int_{0}^{1}\left\|\sum_{i=1}^{N} \Delta\left(\varepsilon_{i} x_{i}: i \in A_{j}\right)\right\|^{\alpha} d t\right\}^{1 / \alpha} & \leqslant 0\left\{\sum_{i=1}^{N} \int_{0}^{1}\left\|\Delta\left(\varepsilon_{i} x_{i}: i \in A_{j}\right)\right\|^{\alpha} d t\right\}^{1 / \alpha}
\end{aligned}
$$

(by symmetrization)

$$
\begin{aligned}
& \leqslant \bullet \theta_{n}\left\{\sum_{i=1}^{N}\left(\sum_{i \in A_{j}}\left\|x_{i}\right\|^{q}\right)^{p / q}\right\}^{1 / \alpha} \\
& \leqslant c \theta_{n}\left\{\sum_{i=1}^{N}\left(\frac{2}{N}\right)^{q / p-1} \sum_{i \in A_{j}}\left\|x_{i}\right\|^{p}\right\}^{1 / \alpha} \\
& \leqslant\left(\frac{2}{N}\right)^{1 / p-1 / \alpha} \Delta \theta_{n} .
\end{aligned}
$$

Hence

$$
\left\{\int_{0}^{1}\left\|\Delta\left(\varepsilon_{1} x_{1}, \ldots, \varepsilon_{n} x_{n}\right)\right\|^{q} d t\right\}^{1 / q} \leqslant c K \theta_{N}+\left(\frac{2}{N}\right)^{1 / p-1 / \alpha} e \theta_{n} .
$$

Thus

$$
\theta_{n} \leqslant \max \left\{\theta_{n}\left(1-\frac{1}{N}\right)^{1 / r}+K \theta_{2} c ; c K \theta_{N}+\left(\frac{2}{N}\right)^{1 / p-1 / q} c \theta_{n}\right\} .
$$

If wo choose $N$ so that

$$
\left(\frac{2}{N}\right)^{1 / p-1 / \alpha}<1
$$

this implies a bound on $\theta_{n}$.

The fact that $\theta_{n}$ is bounded implies that $X \oplus_{N} X$ is type $p$ in the usual way, as in Theorem 6.4.

7. Twisted sums with equal convexity. Since the twisted sum of two Banach spaces may not be convex we may ask what class it does belong to. It turns out that we can give a complete answer to this. We require first the following lemma. We ase the notation of Section 5 . 
LFimma 7.1. Suppose $\mu \geqslant 0$ and $Y$ is a Banach space.

(7.1.1) If $d_{n}(X)=1$ and $d_{n}(Y)=1$, then $o_{n}=O(\log n)$.

(7.1.2) If $\delta_{n}(X) \leqslant n^{\mu}$ and $\delta_{n}(Y)=O\left(n^{\mu}\right)$ (or $\delta_{n}(X)=O\left(n^{\mu}\right)$ and $\left.\delta_{n}(Y) \leqslant n^{\mu}\right)$, then $\gamma_{n}=O\left(n^{\mu} \log n\right)$.

Proof. We prove only (7.1.2) (as the same argument then proves (7.1.1)). If $\delta_{n}(X) \leqslant n^{\mu}$ and $\delta_{n}(Y) \leqslant C n^{\mu}$, then by Theorem 5.2

$$
\frac{\gamma_{m n}}{(m n)^{\mu}} \leqslant \frac{\gamma_{m}}{m^{\mu}}+C \frac{\gamma_{n}}{n^{\mu}}
$$

and hence $\gamma_{n^{k}} n^{-k \mu} \leqslant C k \gamma_{n} n^{-\mu}$ and the result follows easily.

THeorism 7.1. Suppose $X$ and $Y$ are Banach spaces. Then any twisted. sum $Y \oplus_{F} X$ is logconvex.

Proof. Here we have $d_{n}(X)=d_{n}(Y)=1$ for all $n$ and hence $c_{n}$ $\leqslant C(\log n+1)$. Induction on $n$ as in Lemma 3.2 of [6] shows that

$$
\left\|\Delta\left(x_{1}, \ldots, x_{n}\right)\right\| \leqslant M \sum_{k=1}^{n} k_{k}\left\|x_{k}\right\|, \quad x_{1}, \ldots, x_{n} \in X
$$

in this case, for some $M$ independent of $x_{1}, \ldots, x_{n}$.

Suppose $\left\|x_{1}\right\|+\left\|x_{2}\right\|+\ldots+\left\|x_{n}\right\|=1$ and suppose $\left\|x_{1}\right\| \geqslant\left\|x_{2}\right\| \geqslant \ldots$ $\ldots \geqslant\left\|x_{n}\right\|>0$. Let $N_{k}$ be the greatest suffix such that $\left\|x_{N_{k}}\right\|>2^{-k}$ (k $=1,2, \ldots$ ) and let

$$
u_{k_{k}}=\sum_{i=N_{k-1}+1}^{N_{k}} x_{i}, \quad k=1,2, \ldots
$$

(where $N_{0}=0$ ). Suppose $N_{l}=n$. Then

$$
\begin{gathered}
\Delta\left(x_{1}, \ldots, x_{n}\right)=\Delta\left(u_{1}, \ldots, u_{l}\right)+\sum_{k=1}^{l} \Delta\left(x_{N_{k-1}+1}, \ldots, x_{N_{k}}\right), \\
\left\|\sum_{k=1}^{l} \Delta\left(x_{N_{k-1}+1}, \ldots, x_{N_{k}}\right)\right\| \leqslant C \sum_{k=1}^{l}\left(1+\log \left(N_{l k}-N_{l k-1}\right)\right) \sum_{N_{k-1}+1}^{N_{k_{k}}}\left\|x_{i}\right\| \\
\leqslant C+C \sum_{k=1}^{l} \log N_{l} \sum_{N_{k-1}+1}^{N_{k}}\left\|x_{i}\right\| .
\end{gathered}
$$

Clearly, $N_{k^{2}} 2^{-k} \leqslant 1$ so that $N_{k} \leqslant 2 /\left\|x_{i}\right\|$ for $N_{k-1}+1<i \leqslant N_{k}$. Hence

$$
\left\|\sum_{k=1}^{l} \Delta\left(x_{N_{k-1}+1}, \ldots, x_{N_{k}}\right)\right\| \leqslant C+C \log 2+C \sum_{i=1}^{n}\left\|x_{i}\right\| \log \frac{1}{\left\|x_{i}\right\|} .
$$

Also

$$
\begin{aligned}
\| \Delta\left(u_{1}, \ldots, u_{l}\right) & \leqslant M \sum_{i=1}^{l} l_{i}\left\|u_{l k}\right\| \leqslant \frac{M}{\log 2} \sum_{i=1}^{n}\left\|x_{i}\right\| \log \frac{2}{\left\|x_{i}\right\|} \\
& \leqslant M+\frac{M}{\log 2} \sum_{i=1}^{n}\left\|x_{i}\right\| \log \frac{1}{\left\|x_{i}\right\|}
\end{aligned}
$$

Thus

$$
\left\|\Lambda\left(x_{1}, \ldots, x_{n}\right)\right\| \leqslant M+\theta+\theta \log 2+\left(\theta+\frac{M}{\log 2}\right) \sum_{i=1}^{n}\left\|x_{i}\right\| \log \frac{1}{\left\|x_{i}\right\|}
$$

whenever $\sum^{4}\left\|x_{i}\right\|: \ldots:$ 1. ILence for general $x_{1}, \ldots, x_{n}$

$$
\left\|\Delta\left(x_{1}, \ldots, x_{n}\right)\right\| \leqslant B_{1} \sum_{i=1}^{n}\left\|x_{i}\right\|+B_{2} \sum_{i=1}^{n}\left\|x_{i}\right\| \log \frac{S}{\left\|x_{i}\right\|}
$$

where $S^{\prime}=\sum_{i=1}^{n}\left\|x_{i}\right\|$.

Now it easily follows that $Y \oplus_{F} X$ is logconvex for

$$
\begin{aligned}
\left\|\sum_{i=1}^{n}\left(y_{i}, x_{i}\right)\right\| & =\left\|y_{i}-F^{\top}\left(\sum x_{i}\right)\right\|+\left\|\sum x_{i}\right\| \\
& =\left\|\sum_{n}^{\top}\left(y_{i}-W^{\prime}\left(x_{i}\right)\right)\right\|+\left\|\Delta\left(x_{1}, \ldots, x_{n}\right)\right\|+\left\|\sum x_{i}\right\| \\
& \leqslant \sum_{i=1}^{\top}\left\|\left(y_{i}, x_{i}\right)\right\|+B_{1} \sum\left\|x_{i}\right\|+B_{2} \sum\left\|x_{i}\right\| \log \frac{s}{\left\|x_{i}\right\|},
\end{aligned}
$$

and the result follows from the fact that the function

$$
\Phi\left(\xi_{1}, \ldots, \xi_{n}\right)=\sum_{i=1}^{n_{n}} \xi_{i}+\sum_{i=1}^{n} \xi_{i} \log \frac{\sum \xi_{j}}{\xi_{i}}, \quad \xi_{1}, \ldots, \xi_{n} \geqslant 0
$$

is monotone in oach $\xi_{i}$, and $\left\|x_{i}\right\| \leqslant\left\|\left(y_{i}, x_{i}\right)\right\|$.

Remark. If we take $X=Y=l_{x}$ and $F: l_{1} \rightarrow l_{1}$ is given on the finitely non-zero sequences by

$$
F(x)=\left(x_{n} \log \frac{\|x\|}{\left|x_{n}\right|}\right),
$$

then $l_{1} \oplus_{\mu} l_{1}$ contains $l_{m}$ (where as usual $\varphi(x)=x(1+\log (1 / x))$ near zero). (See [8].) This shows that the result of Theorem 7.1. is best possible.

THeorem 7.2. A quasi-Banach space is logeonvex if and only if it is the quotient of a subspace of a twisted sum of two Banach spaces. 
Proof. By Theorem 7.1 such a space must be logconvex. The abovo example generalized to $l_{1}(I)$ for arbitrary index sets $I$ enables one to obtain $l_{\varphi}(I)$ as a subspace of a twisted sum of Banach spaces and hence every logconvex space as a quotient (cf. [19] or the method of Theorem 3.3).

DEEINITION 7.3. We say a quasi-Banach space $X$ is of logtype $p \quad(1 \leqslant p$ $\leqslant 2$ ) if for some constant $C<\infty$ we have

$$
\left\{\int_{0}^{1}\left\|\sum \varepsilon_{i}(t) x_{i}\right\|^{p} d t\right\}^{1 / p} \leqslant C\left(1+\sum\left\|x_{i}\right\|^{p}\left(\log \frac{1}{\left\|x_{i}\right\|}\right)^{p}\right)^{1 / p}
$$

whenever $\left\|x_{1}\right\|^{p}+\ldots+\left\|x_{n}\right\|^{p}=1$.

Remark. In order that $X$ is of logtype $p$ it is sufficient that

$$
\left\{\int_{0}^{1}\left\|\sum \varepsilon_{i}(t) x_{i}\right\|^{p} d t\right\}^{1 / p} \leqslant C^{\prime}\left\{\sum\left\|x_{k}\right\|^{p}(1+\log k)^{p}\right\}^{1 / p} .
$$

To see this arrange $x_{k}$ so that $\left\|x_{k}\right\|$ decreases. Then if $\sum\left\|x_{k}\right\|^{p}=1$, $\left\|x_{k}\right\|^{p}$ $\leqslant k^{-1}$ and hence

$$
\log \frac{1}{\left\|x_{k}\right\|} \geqslant \frac{1}{p} \log k
$$

We will see later that (7.3.1) and (7.3.2) are equivalent; of course, for $p=1$ this is immediate from Section 3 , and for $1<p \leqslant 2$ could be established directly in a similar manner. However our indirect methods also establish this result without difficulty.

Definimion 7.4. A Banach space $X$ is of exact type $p$ if

$$
\left\|x_{1}-x_{2}\right\|^{p}+\left\|x_{1}+x_{2}\right\|^{p} \leqslant 2\left(\left\|x_{1}\right\|^{p}+\left\|x_{2}\right\|^{p}\right), \quad x_{1}, x_{2} \in X .
$$

Remarks. If $p=1$, this is automatic. If $p=2$, it implies that $X$ is a Hilbert space, for in this case

$$
\left\|2 x_{1}\right\|^{2}+\left\|2 x_{2}\right\|^{2} \leqslant 2\left(\left\|x_{1}-x_{2}\right\|^{2}+\left\|x_{1}+x_{2}\right\|^{2}\right)
$$

and hence the parallelogram law holds; then apply a result of Jordan and von Neumann [3]. For $1<p<2$ it is sufficient that $X$ is a quotient of a subspace of an $L_{p}$-space.

THe OREM 7.5. Suppose $X$ and $Y$ are Banach spaces of type $p$ where $1 \leqslant p \leqslant 2$. Suppose that either $X$ or $X$ is of exact type $p$. Then any twisted $\operatorname{sum} Z=Y \oplus_{F} X$ satisfies

$$
\left\{\int_{0}^{1}\left\|\sum_{i=1}^{n} \varepsilon_{i}(t) z_{i}\right\|^{p} d t\right\}^{1 / p} \leqslant O\left\{\sum_{k=1}^{n}\left\|z_{k}\right\|^{p}+\sum\left\|z_{l}\right\|^{p}(\log k)^{p}\right\}^{1 / p}
$$

and hence is of logtype $p$.
Proof. If $X$ is of exact type $p$, then

$$
\left\{\int\left\|\sum \varepsilon_{i}(t) x_{i}\right\|^{p} d t\right\}^{1 / p} \leqslant\left(\sum\left\|x_{i}\right\|^{p}\right)^{1 / p}
$$

for $x_{1}, \ldots, x_{n}$ so that $\delta_{n}(x) \leqslant n^{1 / p-1 / 2}$. Hence Lemma 7.1 implies that

$$
\gamma_{n} \leqslant B n^{1 / p-1 / 2}(\log n+1)
$$

By Lemma 5.3 there exists $\alpha>0$ and $M<\infty$ such that

$$
\left\|\Delta\left(x_{1}, \ldots, \infty_{n}\right)\right\| \leqslant M\left(\sum_{k=1}^{n} k^{\alpha}\left\|x_{k}\right\|^{p}\right)^{1 / p}
$$

for $x_{1}, \ldots, x_{n} \in X$.

Now suppose $\left\|x_{1}\right\|^{p}+\ldots+\left\|x_{n}\right\|^{n}=1$ and $\left\|x_{1}\right\| \geqslant \ldots \geqslant\left\|x_{m}\right\|>0$. Let $N_{n}$ be the greatest suffix such that $\left\|b_{N_{k}}\right\|>2^{-7 s}$ and let

$$
u_{k}(t)=\sum_{N_{k=1}+1}^{N_{k}} \varepsilon_{i}(t) x_{i}, \quad k=1,2, \ldots
$$

Suppose $N_{l}=n$. Then

$$
\begin{aligned}
\Delta\left(\varepsilon_{1} x_{1}, \ldots, \varepsilon_{n} x_{n}\right)=\Delta\left(u_{1}(t), \ldots,\right. & \left.u_{l}(t)\right)+ \\
& +\sum_{k=1}^{N_{k}} \Delta\left(\varepsilon_{N_{l-1}+1} x_{N_{k-1}+1}, \ldots, \varepsilon_{N_{k}} x_{N_{k}}\right) .
\end{aligned}
$$

Now lot

$$
\begin{aligned}
a & =\left\{\int_{0}^{1}\left\|\sum_{k=1}^{l} \Delta\left(\varepsilon_{N_{k-1}+1} x_{N_{k-1}+1}, \ldots, \varepsilon_{N_{k}} x_{N_{k}}\right)\right\|^{p} d t\right\}^{1 / p} \\
& \leqslant B_{2}\left\{\int_{0}^{1} \sum_{l=1}^{l}\left\|\Delta\left(\varepsilon_{N_{k-1}+1}+x_{N_{k-1}+1}, \ldots, \varepsilon_{N_{k}} x_{N_{k}}\right)\right\|^{p} d t\right\}^{1 / p}
\end{aligned}
$$

by the symmotrization argument, where $B_{a}$ is the type $p$ constant of $Y$. Feneo

$$
\begin{aligned}
a & \leqslant B B_{2}\left\{\sum_{k=1}^{l}\left(N_{k}-N_{k-1}\right)^{1-p / 2}\left[\log \left(N_{l k}-N_{k-1}\right)+1\right]^{p}\left(\sum_{i=N_{k-1}+1}^{N_{k}}\left\|x_{i}\right\|^{2}\right)^{p / 2}\right\}^{1 / p} \\
& \leqslant B B_{2}\left\{\sum_{k=1}^{l}\left[\log \left(N_{k}-N_{k-1}\right)+1\right]^{p} \sum_{i=N_{k-1}+1}^{N_{k}}\left\|o_{i}\right\|^{p}\right\}^{1 / p} .
\end{aligned}
$$

6-Studia Math. 69, 


\section{Now observe}

$$
\begin{aligned}
\sum_{m+1}^{n}\left(1+\log 7_{i}\right)^{p} & \geqslant \int_{m}^{n}(1+\log x)^{p} d x \\
& =(n-m)(1+\log n)^{p}-p \int_{m}^{n} \frac{x-m}{x}(1+\log x)^{p-1} d x \\
& \geqslant(n-m)(1+\log n)^{p}-p(n-m)(1+\log n)^{n-1} \\
& \geqslant \frac{1}{2}(n-m)(1+\log n)^{p}
\end{aligned}
$$

provided $n \geqslant n_{0}$ where $n_{0}$ depends on $p$.

Hence there is a constant $o$ such that $c>0$ and

$$
\sum_{l=m+1}^{n}(1+\log k)^{p} \geqslant c(n-m)(1+\log n)^{r}
$$

for all $n, m$. Thus we have

$$
\begin{aligned}
\sum_{N_{k-1}+1}^{N_{k_{k}}}(1+\log j)^{p}\left\|x_{j}\right\|^{p} & \geqslant 2^{-k p} \sum_{N_{k-1+1}}^{N_{k}}(1+\log j)^{p} \\
& \geqslant c 2^{-k p}\left(N_{k}-N_{k-1}\right)\left(1+\log N_{k k}\right)^{p} \\
& \geqslant c 2^{-p}\left(1+\log N_{k k}\right)^{p} \sum_{N_{k-1}+1}^{N_{k}}\left\|x_{j}\right\|^{p} .
\end{aligned}
$$

Thus

$$
a \leqslant 2 e^{-1 / p} B B_{2}\left\{\sum_{k=1}^{n}\left\|x_{k}\right\|^{p}(1+\log k)^{p}\right\}^{1 / p} .
$$

Now we shall show that if

then

$$
b=\left\{\int_{0}^{1}\left\|\Delta\left(u_{\mathbf{1}}(t), \ldots, u_{l}(t)\right)\right\|^{p} d t\right\}^{1 / p},
$$

$$
b \leqslant D\left\{\sum_{k=1}^{n}\left\|x_{k}\right\|^{p}(1+\log 7)^{p}\right\}^{1 / p}
$$

for some $D$ independent of $x_{1}, \ldots, x_{n}$. Wo have

(7.5.1) $\quad b \leqslant M\left\{\int_{0}^{1} \sum_{k=1}^{l} k^{\alpha}\left\|u_{k}(t)\right\|^{p} d t\right\}^{1 / p} \leqslant M B_{3}\left\{\sum_{k=1}^{l} k^{a} \sum_{N_{k-1}+1}^{N_{l_{k}}}\left\|x_{i}\right\|^{p}\right\}^{1 / p}$

where $B_{3}$ is the type $p$ constant of $X$. Hence

$$
b \leqslant M_{1}\left\{\sum_{i=1}^{n}\left\|x_{i}\right\|^{p}\left(\log \frac{2}{\left\|x_{i}\right\|}\right)^{a}\right\}^{1 / 2 p}
$$

(for some constant $M_{1}$ )

$$
\leqslant M_{2}\left(\sum_{i=1}^{n}\left\|x_{i}\right\|^{q}\right)^{1 / p}
$$

where $\frac{1}{2} p<q<p$. Let $\theta=q /(p-q)>1$. Then

$$
\begin{aligned}
& b \leqslant M_{2}\left(\sum_{k= \pm 1}^{n} 7 k^{\alpha / x}\left\|x_{k l}\right\| \|_{k}^{\alpha}-\alpha / p\right)^{1 / p} \\
& \leqslant M_{2}\left(\sum_{k=1}^{n} k_{k=1} \| x_{t}||^{p}\right)^{\alpha / p^{2}}\left(\sum_{k=1}^{n} k^{-\theta}\right)^{1 / p-\alpha / p^{2}} \leqslant I r_{3}\left(\sum_{k=1}^{n} k_{i}\left\|x_{k}\right\|^{p}\right)^{1 / 2} .
\end{aligned}
$$

Combining

$$
a+b \leqslant M M_{1}\left(\sum_{k=1}^{n} z\left\|x_{k}\right\|^{p}\right)^{1 / p}
$$

so that

$$
\left\{\int\left\|\Delta\left(\varepsilon_{1} x_{1}, \ldots, \varepsilon_{n} x_{n}\right)\right\|^{p} d t\right\}^{1 / p} \leqslant M_{4}\left(\sum_{k=1}^{n} k\left\|x_{l l}\right\|^{p}\right)^{1 / p}
$$

and this must hold for any $x_{1}, \ldots, x_{n} \in X$. Returning to (7.5.1) it is clear that (after a symmetrization argument) we may now take $a=1$, and in $(7.5 .2)$

$$
b \leqslant M_{5}\left\{\sum_{i=1}^{n}\left\|x_{i}\right\|^{p} \log \frac{2}{\left\|x_{i}\right\|}\right\}^{\lambda / p} .
$$

By Lemma 3.5

$$
b \leqslant M_{6}\left\{\sum_{k=1}^{n}\left\|x_{k}\right\|^{p}(1+\log k)\right\}^{1 / p}
$$

and combining we now have the estimate

$$
\left\{\int_{0}^{1}\left\|\Delta\left(\varepsilon_{1} x_{1}, \ldots, \varepsilon_{t h} x_{n}\right)\right\|^{p} d t\right\}^{1 / p} \leqslant O\left\{\sum_{k=1}^{n}\left\|x_{k}\right\|^{p}(1+\log k)^{p}\right\}^{1 / p}
$$

We omit the verification this implies the desired property of $Z=X \oplus_{R} X$. Remark. We observe that Theorem 7.5 is best possible, in the sense that each $p, 1 \leqslant p \leqslant 2$, there is a twisted sum $Z_{p}$ of $l_{p}$ and itself and a constant $o>0$ such that if $\xi_{1}^{p}+\ldots+\xi_{n}^{p}=1$ there are $z_{i} \in Z_{p}$, $i=1,2, \ldots, n$ with $\left\|z_{i}\right\|=\xi_{i}$ and

$$
\left\{\int_{0}^{1}\left\|\sum_{i=1}^{n} \varepsilon_{i} z_{i}\right\|^{p} d t\right\}^{1 / p} \geqslant c\left(1+\sum\left\|z_{i}\right\|^{p}\left(\log \frac{1}{\left\|z_{i}\right\|}\right)^{p}\right)^{1 / p} .
$$


Indeed, consider the spaces $Z_{p}$ of [9]. Then $Z_{p}=l_{p} \oplus_{F} l_{p}$ where $F: l_{p}^{0} \rightarrow l_{p}^{0}$ is defined by

$$
F(x)=\left(x_{n} \log \frac{\|x\|}{\left|x_{n}\right|}\right) \text {. }
$$

If $e_{n}$ is the nth basis vector of $l_{p}$, then

$$
\begin{aligned}
\left\|\left(0, \sum \pm \xi_{n} e_{n}\right)\right\| & =\left\|F\left(\sum \pm \xi_{n} e_{n}\right)\right\|+\left\|\sum \pm \xi_{n} e_{n}\right\| \\
& =\left(\sum \xi_{n}^{p}\left(\log \frac{1}{\xi_{n}}\right)^{p}\right)^{1 / p}+\left(\sum \xi_{n}^{p}\right)^{1 / p} \\
& \geqslant\left(1+\sum \xi_{n}^{p}\left(\log \left(\frac{1}{\xi_{n}}\right)\right)^{p}\right)^{1 / p} .
\end{aligned}
$$

Similarly this implies

$$
\left(1+\sum \xi_{n}^{p}\left(\log \left(\frac{1}{\xi_{n}}\right)\right)^{p}\right)^{1 / p} \leqslant C^{\prime}\left(\sum \xi_{n}^{p}(1+\log n)^{p}\right)^{1 / p}
$$

so that (7.3.1) and (7.3.2) are equivalent.

8. Twisted sums of $l_{1}$ and $\boldsymbol{R}$. We now recall two ways of forming a twisted sum $\boldsymbol{R} \oplus_{F} l_{1}$. One method due to the author is by defining $F_{1}: l_{1}^{0}$ $\rightarrow \boldsymbol{R}$ by

$$
F_{1}(x)=\sum_{n=1}^{\infty} \tilde{x}_{n} \log n, \quad x \geqslant 0,
$$

where $\left(\tilde{x}_{n}\right)$ is the decreasing rearrangement of $x$, and

$$
F_{1}(x)=F_{1}\left(x^{+}\right)-F_{1}\left(x^{-}\right)
$$

where $x=x^{+}-x^{-}$where $x^{+} \geqslant 0, x^{-} \geqslant 0$ and $\left|x^{+}\right| \wedge\left|x^{-}\right|=0$; see [6]. The other functional due to Ribe [15] is $F_{2}: l_{1}^{0} \rightarrow \boldsymbol{R}$ given by

$$
F_{2}(x)=\sum_{n=1}^{\infty} x_{n} \log \frac{\|x\|}{\left|x_{n}\right|}
$$

(Actually Ribe uses the equivalent functional

$$
\left.\tilde{\boldsymbol{F}}_{2}(x)=\sum_{n=1}^{\infty} x_{n} \log \frac{1}{x_{n}}+\left(\sum_{n=1}^{\infty} x_{n}\right) \log \left(\sum_{n=1}^{\infty} x_{n}\right) .\right)
$$

\|\|$_{1}$ and \|\|$_{2}$ denote the induced norms on $\boldsymbol{R} \oplus l_{1}$. Then if $e_{n}$ is the $n$th basis vector of $l_{1}$ and $t_{n} \geqslant 0(n=1,2, \ldots, N)$

$$
\left\|\left(0, \sum_{i=1}^{N} t_{n} e_{n}\right)\right\|_{1}=\sum_{n=1}^{N}\left|t_{n}\right|+\sum_{n=1}^{N} \tilde{t}_{n} \log n
$$

and

$$
\left\|\left(0, \sum_{n=1}^{N} t_{n} e_{n}\right)\right\|_{2}=\sum_{n=1}^{N}\left(t_{n}\right)+\sum_{n=1}^{\infty} t_{n} \log \frac{\left(\sum t_{k}\right)}{t_{n}} .
$$

Thus we see for both $F_{1}$ and $F_{2}$ we have examples to show

THEOREM 8.1. There is a twisted sum of $\boldsymbol{R}$ and $l_{\mathbf{l}}$ where galb is $l_{\varphi}$.

In view of this we remark that these two twisted sums are not projectively equivalent ([9]) in the sense that there is no isomorphism $\boldsymbol{S}: \boldsymbol{R} \oplus_{\boldsymbol{F}_{1}} l_{\boldsymbol{1}}$ $\rightarrow \boldsymbol{R} \oplus_{N_{2}} l_{l_{1}}$ such that the diagram

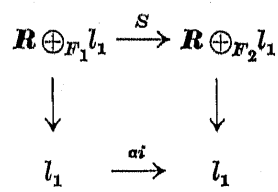

commutes where $\alpha \neq 0$. For (see [9]), projective equivalence implies the existence of $a \neq 0, N<\infty$ and a linear map $t: l_{1}^{0} \rightarrow \boldsymbol{R}$ so that

$$
\left|F_{1}(a x)-F_{2}(x)-t(x)\right| \leqslant N\|x\|, \quad x \in l_{\mathbf{1}}^{0} .
$$

Since $F_{1}\left(e_{n}\right)=F_{2}\left(e_{n}\right)=0$ for all $n$, this would imply $t\left(e_{n}\right)$ bounded so that $t$ is continuous and

$$
\left|F_{1}(a x)-F_{2}(x)\right| \leqslant(N+\|t\|)\|x\|, \quad x \in l_{1}^{0} .
$$

Now by Lemma 3.5 we see the only possible value of $a$ is $a=1$. Thus

$$
\left|F_{1}(x)-F_{2}(x)\right| \leqslant(N+\|t\|)\|x\| .
$$

Now let $x_{N}=\sum_{n=1}^{N} \frac{1}{n} e_{n}$.

$$
\begin{gathered}
F_{1}\left(x_{N}\right)=\sum_{n=1}^{N} \frac{\log n}{n}, \\
F_{2}\left(x_{N}\right)=\sum_{n=1}^{N} \frac{1}{n}\left(\log n+\log S_{N}\right)
\end{gathered}
$$

where $S_{N}=\sum_{n=1}^{N} \frac{1}{n}$. Hence

$$
F_{2}\left(x_{N}\right)-F_{1}\left(x_{N}\right)=S_{N} \log S_{N}
$$

while $\left\|x_{N}\right\|=S_{N} \rightarrow \infty$ and so we have a contradiction. 
To conclude this short section we consider the following:

DEEINITION 8.2. An operator $\tilde{T}: l_{1} \rightarrow l_{1}$ is liftable if for every twisted sum $\boldsymbol{R} \oplus_{F} l_{1}$ there is a map $\tilde{T}: l_{1} \rightarrow \boldsymbol{R} \oplus_{T} l_{x}$ such that the diagram

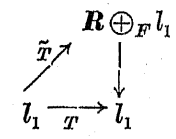

commutes.

THEOREM 8.3. Let $T: l_{1} \rightarrow l_{1}$ be given by $T e_{n}=d_{n} e_{n}$ where $\|T\|$ $=\sup \left|\boldsymbol{d}_{b}\right|<\infty$. Then the following are equivalent:

(a) $T$ is liftable.

(b) For some $\tau<\infty, \sum_{n=1}^{\infty} \exp \left(-\tau /\left|d_{n}\right|\right)<\infty$.

(c) $\left[d_{n}\right] \in c_{0}$ and if $d_{n}^{*}$ is the decreasing re-arrangement of $\left(d_{n}\right)$, then $\left\{d_{n}^{*} \log n\right\}$ is bounded.

(d) $\boldsymbol{T}\left(l_{1}\right) \subset l_{\infty}$.

(e) For any logeonvex space $X$ and quotient map $q: X \rightarrow l_{\mathbf{1}}$ there is an operator $S: l_{1} \rightarrow X$ such that $q S=T$.

Proof. It clearly suffices to consider the case $a_{n} \geqslant 0$. Note first that if $d_{n} \rightarrow 0$, then there is a projection $P$ onto a subspace isomorphic to $l_{1}$ such that $\dot{P}=S T$ for some bounded $S$. Then $T$ is liftable so is $P$ and this clearly contradicts the fact that $\left(\boldsymbol{R}, l_{1}\right)$ does not split. Here we may assume $d_{n} \rightarrow 0$ and then we may suppose $\left\{d_{n}\right\}$ decreasing.

(a) $\Rightarrow$ (c). Oonsider

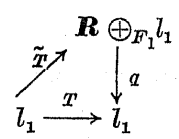

Supposo $\tilde{\boldsymbol{T}} e_{n}=\left(c_{n}, d_{n} e_{n}\right)$. Then

and

$$
\left\|\tilde{\boldsymbol{T}} e_{n}\right\|_{1}=\left|c_{n}\right|+\left|d_{n}\right| \leqslant\|\tilde{\boldsymbol{T}}\|
$$

$$
\begin{aligned}
\left\|\tilde{T}\left(e_{1}+\ldots+e_{n}\right)\right\|_{1} & =\sum_{k=1}^{n} d_{k_{k}}+\left|\sum_{k=1}^{n} e_{k}-\sum_{i=1}^{n} d_{l_{k}} \log k_{k}\right| \\
& \geqslant \sum_{k=1}^{n} d_{k_{k}}+\sum_{k=1}^{n} d_{k} \log k-\sum_{k=1}^{n} c_{l_{k}} \\
& \geqslant n d_{n} \log n-n\|\tilde{T}\| .
\end{aligned}
$$

Hence

$$
d_{n} \log n \leqslant 2\|\tilde{T}\|
$$

(c) $\Rightarrow$ (b). If $d_{n} \leqslant b(\log n)^{-1}, n \geqslant 2$, then if $\tau>b$

$$
\exp \left(-\frac{\tau}{d_{n}}\right) \leqslant n^{-(\tau / b)} \text {. }
$$

(b) $\Rightarrow$ (d). Suppose $\left|t_{1}\right|+\ldots+\left|t_{n}\right| \leqslant 1$. Then

$$
\begin{aligned}
\left\|T\left(t_{1} e_{1}+\ldots+t_{n} e_{n}\right)\right\|_{p}^{*}= & \sum_{i=1}^{n} d_{i}\left|t_{i}\right|+\sum_{i=1}^{n} d_{i}\left|t_{i}\right| \log \frac{\sum_{j=1}^{n} d_{i}\left|t_{j}\right|}{d_{i}\left|t_{i}\right|} \\
= & S+S \log S-\sum_{i=1}^{n} d_{i}\left|t_{i}\right| \log d_{i}- \\
& -\sum_{i=1}^{n} d_{i}\left|t_{i}\right| \log \left|t_{i}\right|
\end{aligned}
$$

where $S=\sum_{i=1}^{n} d_{i}\left|t_{i}\right| \leqslant\|T\|$. Also $-d_{i} \log d_{i} \leqslant e^{-1}$. Hence

$$
\left\|T\left(t_{1} e_{1}+\ldots+t_{n} e_{n}\right)\right\|_{p}^{*} \leqslant\|T\|+\|T\| \log \|T\|+e^{-1}+\sum_{i=1}^{n} d_{i}\left|t_{i}\right| \log \frac{1}{\left|t_{i}\right|} .
$$

Now suppose $\xi_{1}, \ldots, \xi_{n} \geqslant 0$ are chosen to maximize $\psi\left(\xi_{1}, \ldots, \xi_{n}\right)$

$=\sum_{i=1}^{n} d_{i} \xi_{i} \log \frac{1}{\xi_{i}}$ subject to $\xi_{1}+\ldots+\xi_{n}=1$.

Then there is a Lagrange multiplier $\lambda$ such that if $\xi_{i} \neq 0$,

i.e.,

$$
\lambda_{i} \log \frac{1}{\xi_{i}}-d_{i}=\lambda
$$

so that

$$
\log \frac{1}{\xi_{i}}=1+\frac{\lambda}{d_{i}}
$$

$$
\xi_{i}=e^{-\left(1+\lambda / d_{i}\right)} .
$$

Lot $A=\left\{i: \xi_{i}>0\right\}$. Thon

$$
\begin{gathered}
\sum_{i \in A} e^{-\left(1+\lambda / d_{i}\right)}=1 \\
\psi\left(\xi_{1}, \ldots, \xi_{n}\right)=\sum_{i \in A} d_{i} e^{-\left(1+2 / d_{i}\right)}\left(1+\lambda / a_{i}\right) \leqslant\|T\|+\lambda .
\end{gathered}
$$

Now

$$
\sum_{i \in i} e^{-\lambda / d_{i}}=0
$$


Hence

$$
\sum_{i=1}^{\infty} e^{-\lambda / d_{i}} \geqslant e
$$

Since for some $\tau<\infty$

$$
\sum e^{-\lambda / d_{i}}<\infty,
$$

there exists $\lambda_{0}$ such that $\lambda>\lambda_{0}$ implies

$$
\sum_{i=1}^{\infty} e^{-\lambda / d_{i}}<e
$$

Thus $\gamma \leqslant \lambda_{0}$ and so

$$
\left\|T\left(t_{1} e_{1}+\ldots+t_{n} e_{n}\right)\right\|_{\varphi}^{*} \leqslant\|T\|(2+\log \|T\|)+e^{-1}+\lambda_{0} .
$$

Hence $T$ maps $l_{1}$ into $l_{\varphi}$.

(d) $\Rightarrow(e)$.

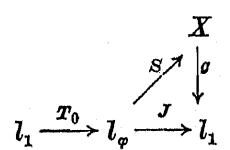

$T$ factors $T=J T_{0}$ where $J: l_{\varphi} \rightarrow l_{1}$ is the inclusion map. The existence of a lift $S$ follows from the fact that $X$ is logconvex.

(c) $\Rightarrow$ (a). Theorem 7.1.

9. Orlicz sequence spaces. We recall that an $F$-space $X$ is a $\mathscr{K}$-space if $(\boldsymbol{R}, X)$ splits $([6],[8])$. In this section we classify completely those locally banded Orlicz sequence spaces $l_{f} \subset l_{1}$ which are $\mathscr{K}$-spaces. It is known ([6]) that $l_{p}$ is a $\mathscr{K}$-space if $0<p<1$ and fails to be a $\mathscr{K}$-space if $p=1$.

We shall suppose throughout that $f$ is a twice-differentiable strictly increasing Orlicz function with $f(1)=1$ such that $x f(x)$ is convex (cf. [5]); these assumptions may be made without loss of generality. We also suppose that $f$ satisfies the $\Delta_{2}$-condition, i.e. for some $K$

We define

$$
f(2 x) \leqslant K f(x), \quad 0 \leqslant x<\infty .
$$

$$
\begin{aligned}
& a_{f}=\sup \left\{p: \exists M, f(a x) \leqslant M a^{p} f(x), 0<a, x<1\right\}, \\
& \beta_{f}=\inf \left\{p: \exists M, f(a x) \geqslant M a^{p} f(x), 0<a, x<1\right\} .
\end{aligned}
$$

Since $l_{f}$ is locally bounded, $\alpha_{f}>0$, and the $\Delta_{2}$-condition implies $\beta_{f}<\infty$. Since $l_{f} \subset l_{1}$, we shall suppose

for some $M$.
Now let $h: \boldsymbol{R} \rightarrow \boldsymbol{R}$ be defined by

$$
\begin{aligned}
& h(x)=x \int_{x}^{1} \frac{f(t)}{t^{2}} d t, x>0, \\
& h(0)=0, \\
& h(x)=-h(-x), \quad x<0 .
\end{aligned}
$$

LiEMara 9.1. Th has the following properties:

(i) $h$ is continuous, and twioe differentiable for $x \neq 0$.

(ii) $h^{\prime \prime}(u) \leqslant 0, u \neq 0$.

(iii) If $u+v+w=0$,

$$
h(u)+h(v)+h(w) \leqslant 2(f(|u|)+f(|v|)+f(|w|)) .
$$

(iv) If $0 \leqslant a \leqslant 1$ and $x \in \boldsymbol{R}$,

$$
|h(a x)-a h(x)| \leqslant f(x) .
$$

Proof. (i) For continuity at 0 , observe if $0<x<1$

$$
h(x) \leqslant \infty \int_{x}^{1} \frac{M}{t} d t \leqslant M x \log \frac{1}{x} .
$$

The other assertion is clear.

$$
\text { (ii) } \begin{aligned}
h^{\prime}(u) & =\int_{a}^{1} \frac{f(t)}{t^{2}} d t-\frac{f(u)}{u} u>0, \\
h^{\prime \prime}(u) & =-\frac{f^{\prime}(u)}{u} \leqslant 0 .
\end{aligned}
$$

(iii) Suppose without loss of generality $u>0, v>0$ and $w=-(u+v)$. Since $h^{\prime \prime} \leqslant 0$,

so that

$$
h(u+v) \leqslant h(u)+h(v) \leqslant 2 h\left(\frac{1}{2}(u+v)\right)
$$

$$
0 \leqslant h(u)+h(v)+h(w) \leqslant 2 h\left(\frac{1}{2}(u+v)\right)-h(u+v),
$$

$2 h\left(\frac{1}{2}(u+v)\right)-h(u+v)=(u+v) \int_{\frac{u}{u}(u+v)}^{u+v} \frac{f(x)}{x^{2}} d x \leqslant 2 f(u+v)$.

Hence

$$
h(u)+h(v)+h(w) \leqslant 2 f(|w|) \leqslant 2(f(|u|)+f(|v|)+f(|w|)) .
$$


(iv) For $x>0$.

$h(a x)-a h(x)=a x \int_{a x}^{x} \frac{f(t)}{t^{2}} d t \leqslant a x f(x) \int_{a x}^{x} \frac{1}{t^{2}} d t=f(x)(1-a) \leqslant f(x)$.

Lemars 9.2. Suppose for some $B<\infty$ we have for $0 \leqslant x \leqslant 1$.

$$
h(x) \leqslant B f(x) .
$$

Then $\beta_{f}<1$.

Proof. Let $O_{f} \subset O[0,1]$ bo defined by

where

$$
O_{f}=\overline{c o}\left\{f_{t}: 0<t \leqslant 1\right\}
$$

$$
f_{t}(x)=\frac{f(t x)}{f(t)}
$$

(ef. [5], [10]). Since $l_{f} \subset l_{1} ; a_{f} \leqslant 1$. If $\beta_{f} \geqslant 1$, then $x \in C_{f}$ ([10]).

$$
\int_{x}^{1} \frac{f(t)}{t^{2}} d t \leqslant \frac{f(x)}{x} .
$$

and if $0<s \leqslant 1$

$$
\begin{aligned}
\int_{x}^{1} \frac{f_{s}(t)}{t^{2}} d t & =\int_{x}^{1} \frac{f(s t)}{t^{2} f(s)} d t=\int_{s x}^{f} \frac{s f(u)}{u^{2} f(s)} d u \leqslant \frac{s}{f(s)} \int_{s: x}^{1} \frac{f(u)}{u^{2}} d u \\
& \leqslant B \frac{s}{f(s x)} \frac{f(s x)}{s x}=\frac{B f_{s}(x)}{x}, \quad 0<x \leqslant 1 .
\end{aligned}
$$

Hence, if $g \in O_{f}$

$$
\int_{x}^{1} \frac{g(t)}{t^{2}} d t \leqslant B \frac{g(x)}{x}, 0<\infty \leqslant 1 .
$$

In particular if $\beta_{f} \geqslant 1$, we may let $g(t)=t$

$$
\int_{x}^{1} \frac{1}{t} d t \leqslant B, \quad 0<x \leqslant 1
$$

and this contradiction shows $\beta_{f}<1$.

THeORem 9.3. Suppose $f$ is a Orliaz function satisfying the $\Delta_{2}$-condition and that $l_{f}$ is locally bounded and contained in $l_{1}$. Then $l_{f}$ is a $\mathscr{K}$-space if and only if $\beta_{f}<1$.
Proof. If $\beta_{f}<1, l_{f}$ is a $\mathscr{K}$-space [6]. Conversely, suppose $l_{f}$ is a $\mathscr{K}$-space. We define

$$
H: l_{f}^{0} \rightarrow \boldsymbol{R}
$$

(where $l_{f}^{0}$ is the finitely non-zero sequences in $l_{f}$ ) by

$$
H(\infty)=\sum_{i=1}^{\infty} h\left(x_{i}\right) \quad \text { if } \quad \sum_{i=1}^{\infty} f\left(\left|x_{i}\right|\right)=1
$$

and extend so that

$$
H(a x)=a H(x), \quad a \in \boldsymbol{R} .
$$

We first assert that $H: l_{f}^{0} \rightarrow \boldsymbol{R}$ is quasilinear. To see this we show that if $u, v, w \in l_{f}^{0}$

and

$$
u+v+w=0
$$

then

Indeed,

$$
\|u\|+\|v\|+\|w\| \leqslant 1
$$

$$
|H(u)+H(v)+H(w)| \leqslant 9 .
$$

$$
\left|H(u)-\sum_{i=1}^{\infty} h\left(u_{i}\right)\right| \leqslant 1
$$

and similarly for $v, w$ while

$$
\left|\sum_{i=1}^{\infty} h\left(u_{i}\right)+h\left(v_{i}\right)+h\left(w_{i}\right)\right| \leqslant 6 .
$$

Now $l_{f}$ is a $\mathscr{K}$-space so that there a linear map $\psi: l_{f}^{0} \rightarrow \boldsymbol{R}$ such that

$$
\sup _{\|x\|<1}|H(x)-\psi(x)|<\infty .
$$

Thus, $\left\{\psi\left(e_{n}\right): n=1,2, \ldots\right\}$ is bounded since $H\left(e_{n}\right)=0$ and so $\psi$ is continuous. \#onco,

$$
|H(x)| \leqslant L\|x\|, \quad x \in l_{\rho}^{0},
$$

for some $L<\infty$.

Suppose $0<\xi \leqslant 1$; chooso $n$ so that $n \in N$ and $\frac{1}{2}<n \xi \leqslant 1$. Choose $\eta$ so that $f(\eta)=1-n \xi$ and lot

Then $\|x\|=1$ and

$$
x=\xi\left(e_{1}+\ldots+e_{n}\right)+\eta e_{n+1} \text {. }
$$

$$
H(x) \geqslant n \xi \int_{\xi}^{1} \frac{f(t)}{t^{2}} d t \geqslant \frac{1}{2} \frac{\xi}{f(\xi)} \int_{\xi}^{1} \frac{f(t)}{t^{2}} d t .
$$


Hence,

$$
\int_{\xi}^{1} \frac{f(t)}{\xi} d t \leqslant 2 L \frac{f(\xi)}{\xi}, \quad 0<\xi \leqslant 1,
$$

and so by Lemma $9.2, \beta_{f}<1$.

Suppose now $f$ is submultiplicative at 0 . Then we say $X$ is $f$-oonvex if $\sum f\left(\left\|x_{i}\right\|\right)<\infty$ implies $\sum x_{i}$ converges, i.e., $X$ is galbed by $l_{f}$.

CoROLLARY 9.4. Suppose $f$ is submultiplicative at 0 ; then every twisted sum of $\boldsymbol{R}$ and a f-convex space is also f-convex if and only if

$$
\beta_{f}=\lim _{x \rightarrow 0} \frac{\log f(x)}{\log x}<1 \text {. }
$$

Proof. Observe that $l_{f}(I)$ (for an index set $I$ ) is projective among $f$-convex, i.e.

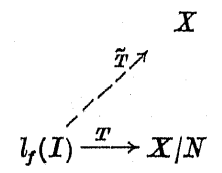

Hence if every twisted sum of $\boldsymbol{R}$ and $l_{f}$ is $f$-convex, then $l_{f}$ is a $\mathscr{K}$-space. Conversely, suppose $l_{f}$ is a $\mathscr{K}$-space and $X$ is any $f$-convex space and $\boldsymbol{Y}=\boldsymbol{R} \oplus_{\boldsymbol{B}} X$ is a twisted sum of $\boldsymbol{R}$ and $X$. Then there is a quotient map $T: l_{f}(I) \rightarrow X$ for some index set $I$. Now $l_{f}(I)$ is also a $\mathscr{K}$-space (this is easy to show) and so there is a lift $\tilde{T}: l_{f}(I) \rightarrow Y$. If $\tilde{T}$ fails to be surjective $\bar{Y}$ (splits, while if $\tilde{T}$ is surjective, $Y$ is $f$-convex.

Remark. In this case $\beta_{f}<1$ implies $f(x) \geqslant c x^{p}$ for some $p<1$, and $c>0$ for all $0 \leqslant \infty \leqslant 1$

10. Locally convex $\mathscr{K}$-spaces. Let $X$ be a metrizable locally convex space. Let $\|\cdot\|_{n}$ be a sequence of semi-norms on $X$ which define the topology of $X$ and such that

$$
\|x\|_{n} \leqslant\|x\|_{n+1}, \quad n \in \boldsymbol{N} .
$$

Define a map $F: X \rightarrow \boldsymbol{R}$ be quasilinear if for some $n \in \boldsymbol{N}, K<\infty$

$$
\begin{gathered}
F^{\prime}(t x)=t F^{\prime}(x), \quad t \in \boldsymbol{R}, x \in X, \\
\left|F^{\prime}(x+y)-F^{\prime}(x)-W^{\prime}(y)\right| \leqslant K\left(\|x\|_{n}+\|y\|_{n}\right)
\end{gathered}
$$

if $F$ is quasilinear we define $\boldsymbol{R} \oplus_{F} X$ to be the space $\boldsymbol{R} \oplus X$ equipped with the quasi-semi-norms

$$
\|(t, x)\|_{m}^{*}=|t-F(x)|+\|x\|_{m}, \quad m \geqslant n .
$$

Then if $q: \boldsymbol{R} \oplus_{F} X \rightarrow X$ is given by

$$
q(t, x)=x,
$$

$q$ is a quotient map and $q^{-1}(0)=\{(t, 0), \dot{t} \in \boldsymbol{R}\}$. Thus we have a short exact sequence $0 \rightarrow \boldsymbol{R} \rightarrow \boldsymbol{R} \oplus_{H} X \rightarrow X \rightarrow 0$ and $\boldsymbol{R} \oplus_{F} X$ is a twisted sum of $\boldsymbol{R}$ and $X$. It is easy to show that if $X$ is complete, then so is $\boldsymbol{R} \oplus_{F} X$ (since it is such a twisted sum).

The twisted sum $\boldsymbol{R} \oplus_{n} \boldsymbol{X}$ will split if and only if there is a linear $\operatorname{map} \psi: \mathcal{X} \rightarrow \boldsymbol{R}$ such that

$$
\left|W^{\prime}(x)-\psi(x)\right| \leqslant M\|x\|_{m}, \quad x \in X
$$

for some $m \in N$ and $M<\infty$.

THDORIEM 1.0.1. Let $X$ be a Fréchet space (complete metrizable locally convex space) and suppose an $F$-spaoe $Y$ is a twisted sum of $\boldsymbol{R}$ and $X$. Then there exists a quasilinear map $F: X \rightarrow \boldsymbol{R}$ such that $Y$ is isomorphio to $\boldsymbol{R} \oplus_{F} X$ (as a twisted sum).

Proof. It is convenient to write $Y=\boldsymbol{R} \oplus X$ algebraically so that the quotient map $q: X \rightarrow X$ is given by $q(t, x)=x$.

Let $\left\{V_{n}: n \in \boldsymbol{N}\right\}$ be a base of balanced neighborhoods of 0 such that $V_{n+1}+V_{n+1} \subset V_{n}$ for all $n$ and $V_{1} \cap \boldsymbol{R e}$ is bounded where $e=(1,0)$. Let $! \cdot !_{n}$ be the Minkowski functional of $V_{n}$. Then we have

$$
\begin{gathered}
!(t, x) !_{n} \leqslant !(t, x) !_{n+1}, \quad t \in \boldsymbol{R}, x \in X, \\
!(s+t, x+y) !_{n} \leqslant !(s, x) !_{n+1}+!(t, x) !_{n+1}, \\
!(1,0) !_{n}=\alpha_{n} \quad \text { where } \quad \alpha_{1}>0 \text { and } \alpha_{n} \uparrow \infty .
\end{gathered}
$$

Also since $q$ is open, there exist increasing sequences $\{m(n)\}$ and $\left\{\beta_{n}\right\}$ such that

(10.1.4) For $x \in X$ there exists $t_{n} \in \boldsymbol{R}$

$$
!\left(t_{n}, \infty\right) !_{n} \leqslant \beta_{n}\|x\|_{m(n)} .
$$

In view of (10.1.4) thero is a map $F_{n}: X \rightarrow \boldsymbol{R}$ such that $F(t x)=t F^{\prime}(x)$, $t \in \boldsymbol{R}$ and

$$
!\left(T_{n}(x), x\right) !_{n} \leqslant \beta_{n}\|x\|_{m(n)}
$$

Now if $n>p>1$

$$
\begin{aligned}
\left|F_{n}(x)-F_{p}(x)\right| & =\alpha_{p-1}^{-1} !\left(F_{n}(x)-F_{p}(x), 0\right) !_{p-1} \\
& \leqslant \alpha_{p-1}^{-1}\left(!\left(F_{n}(x), x\right) !_{p}+!\left(F_{p}(x), x\right) !_{p}\right) \\
& \leqslant \alpha_{p-1}^{-1}\left(\beta_{n}+\beta_{p}\right)\|x\|_{m(n)} .
\end{aligned}
$$


Also if $n \geqslant 3$ and $x, y \in X$

(10.1.6) $\quad !\left(\mathbb{F}_{n}(x)+\boldsymbol{F}_{n}(y), x+y\right) !_{n-1} \leqslant \beta_{n}\left(\|x\|_{m(n)}+\|y\|_{m(n)}\right)$.

(10.1.7) $\quad !\left(\boldsymbol{F}_{n-1}(x+y), x+y\right) !_{n-1} \leqslant \beta_{n-1}\left(\|x\|_{m(n)}+\|y\|_{m(n)}\right)$.

Hence combining (10.1.6) and (10.1.7) with (10.1.2)

$$
a_{n-2}\left|F_{n}(x)+F_{n}(y)-F_{n}(x+y)\right| \leqslant\left(\beta_{n}+\beta_{n-1}\right)\left(\|x\|_{m(n)}+\|y\|_{m(n)}\right) .
$$

Thus

$$
\left|F_{n}(x)+F_{n}(y)-F_{n}(x+y)\right| \leqslant 0_{n}\left(\|x\|_{m(n)}+\|y\|_{m(n)}\right) .
$$

In particular $F \equiv F_{3}$ in quasilinear, and for $n \geqslant 3$

$$
\left|F_{n}(x)-F^{\prime}(x)\right| \leqslant D_{n}\|x\|_{m(n)}
$$

Thus

$$
\begin{aligned}
!(u, x) !_{n} & \leqslant \alpha_{n+1}\left|u-F_{n}(x)\right|+\beta_{n+1} \mid\|x\|_{m(n+1)} \\
& \leqslant \alpha_{n+1}|u-F(x)|+\left(\alpha_{n+1} D_{n}+\beta_{n+1}\right)\|x\|_{m(n+1)} \\
& \leqslant A_{n}\left(|u-F(x)|+\|x\|_{m(n+1)} .\right.
\end{aligned}
$$

Hence the identity $i: \boldsymbol{R} \oplus_{F} X \rightarrow Y$ is continuous. By the closed graph theorem $i$ is an isomorphism.

Tweorem 10.2. Any nuclear Fréchet space is a $\mathscr{K}$-space.

Proof. If $X$ is a nuclear Fréchet space and $F: X \rightarrow \boldsymbol{R}$ is quasilinear, then there is a Hilbertian semi-norm $\|\cdot\|_{n}$ on $X$ such that

$$
\left|F^{\prime}(x+y)-F(x)-F(y)\right| \leqslant\|x\|_{n}+\|y\|_{n}
$$

Since a Hilbert space is a $\mathscr{K}^{\prime}$-space $([6])$, there is a linear map $\psi: X \rightarrow \boldsymbol{R}$ such that

$$
|F(x)-\psi(x)| \leqslant M\|x\|_{n}
$$

Let $\left(a_{m n}\right)$ be a matrix with non-negative entries such that $a_{m+1, n} \geqslant a_{m, n}$ for all $n$ and for each $n$ there exists $n$ with $a_{m n}>0$. Then the Köthe soquence space $l_{1}\left[a_{m n}\right]$ is the space of sequences $\left(x_{n}\right)$ such that

$$
\|x\|_{m}=\sum_{n=1}^{\infty}\left|a_{m n}\right|\left|x_{n}\right|<\infty, \quad m=1,2, \ldots
$$

THEOREM 10.3. $l_{1}\left[a_{m n}\right]$ is a $\mathscr{K}$-space if and only if given $m \in \mathbf{N}$ there exists $\tau<\infty$ and $r>m$ with

$$
\sum_{n=1}^{\infty} \exp \left(-\tau \frac{a_{r n}}{a_{m n}}\right)<\infty \quad(0 / 0=\infty)
$$

Proof. If (10.3.1) fails, there exists $m$ such that for all $r>m$ and $\tau<\infty$

$$
\sum_{n=1}^{\infty} \exp \left(-\tau \frac{a_{r n}}{a_{m n}}\right)=\infty .
$$

Define $F: l_{1}^{0}\left[a_{m n}\right] \rightarrow \mathbb{R}$ by

$$
I^{\prime}(x)=\sum_{n=1}^{\infty} a_{m n} x_{n} \log \frac{\|x\|_{m}}{a_{m n}\left|x_{n}\right|}
$$

(for finitely non-zoro sequonces). If $\boldsymbol{R} \oplus_{m} l_{1}^{0}\left[a_{m n}\right]$ splits then there is a linear map $\psi: l_{1}^{0}\left[a_{m n}\right] \rightarrow \boldsymbol{R}$ stuch that

$$
|F(x)-\psi(x)| \leqslant K\|x\|_{r}
$$

for some $r>m$. This implies

and so

$$
\psi\left(e_{n}\right) \leqslant K a_{r_{n}}
$$

so that

$$
|\psi(x)| \leqslant K\|x\|_{r}
$$

Flence

$$
\left|W^{\prime}(x)\right| \leqslant 2 K\|x\|_{r}
$$

$$
\sum_{n=1}^{\infty} a_{m n}\left|x_{n}\right| \log \frac{\|x\|_{m}}{a_{m n}\left|a_{n}\right|} \leqslant 2 K \sum_{n=1}^{\infty} a_{r n}\left|x_{n}\right| .
$$

This means the diagonal map $\left\{x_{n}\right\} \rightarrow\left\{d_{n} x_{n}\right\}$ maps $l_{1}$ into $l_{p}$ where

$$
a_{n}=\frac{a_{m n}}{a_{r n}} \quad\left(=0 \text { if } a_{r n}=0\right) .
$$

Hence by Theorem 8.3 we have contradicted (10.3.2).

Oonversely if (10.3.1) holds and $F$ is quasilinear, then if

$$
\left|F^{\prime}(x+y)-W^{\prime}(x)-F^{\prime}(y)\right| \leqslant K\left(\|x\|_{m}+\|y\|_{m}\right),
$$

choose $r>m$ to satisfy (10.3.1). Let

$$
d_{n}=\frac{a_{m n}}{a_{m n}}
$$

Then $D:\left\{x_{n}\right\} \rightarrow\left\{d_{n} x_{n}\right\}$ is liftable. If we define $G: l_{1} \rightarrow \boldsymbol{R}$ by

$$
G(x)=F\left(\left\{a_{m n}^{-1} x_{n}\right\}\right) \quad(1 / 0=0 !) .
$$


$G$ is quasilinear on $l_{1}$ and hence there is a linear map $\tilde{D}$

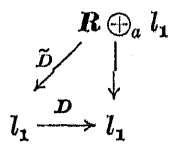

If $\tilde{D} x=(\psi(x), D x)$, then

$$
|\psi(x)-G(D x)| \leqslant O\|x\|, \quad x \in l_{1} .
$$

Hence

$$
\left|\psi\left\{a_{r n} x_{n}\right\}-W^{\prime}(x)\right| \leqslant O\|x\|_{r}, \quad x \in l_{1}\left[a_{m n}\right]
$$

so that $\boldsymbol{R} \oplus_{F} l_{1}\left[a_{m_{n}}\right]$ splits.

Remark. Oondition (10.3.1) is thus a topological invariant of $l_{1}\left[a_{m n}\right]$.

\section{References}

[1] P. Enflo, J. Lindenstrauss and G. Pisier, On the three space problem, Math. Scand. 36 (1975), 199-210.

[2] T. Figiel, J. Lindenstrauss and V. D. Milman, The dimension of almost . spherical sections of convex bodies, Acta Math. 139 (1977), 53-94.

[3] P. Jordan and J. von Neumann, On inner products in linear metrio spaees, Ann. Math. 36 (1935), 719-723.

[4] J. P. Kahane, Some random series of functions, Heath Mathematical Monographs, 1968.

[5] N. J. Kalton, Orlicz sequences without local convexity, Math. Proc. Cambridge Philos. Soc. 81 (1977), 253-277.

[6] - The three space problem for locally bounded F-8paces, Comp. Math. 37 (1978), 243-276.

[7] - Operators on $L_{p}, 0<p<1$, Trans. Amer. Math. Soc. 259 (1980), 319-355.

[8] N. J. Kalton and N. T. Peck, Quotients of $L_{p}(0,1), 0<p<1$, Studia Math. 64 (1978).

[9] -, - Twisted sums of sequence spaces and the three space problem, Trans. A.mer. Math. Soc. 255 (1979), $1-30$.

[10] J. Lindenstrauss and L. Tzafriri, On Orlicz sequenoe space III, Israel J. Math. 14 (1973) 368-389.

[11] -, - Olassical Banach Spaces, Vol. I Sequence Spaces, Springer, 1977.

[12] B. Maurey, Théorèmes des factorization pour les opérateurs lineaires a valeurs dans les espaces $L^{p}$, Asterisque 11 (1974), 1-163.

[13] B. Maurey and G. Pisiex, Series de variables aléatoires vectorielles independantes et propriétés geométriques des espaces de Banaoh, Studia Math. 58 (1976), 45-90.

[14] E. M. Nikisin, Resonance theorems and expansions in eigenfunctions of the Laplace operator, Izv. Akad. Nauk. U.S.S.R. Math. 36 (1972), 795-813 (= Math. U.S.S.R. Izv. 6 (1972), 788-806).
[15] M. Ribe, Dxamples for the nonlocally convex three-space problem, Proc. Amer. Math. Soc. 79 (1979), 351-355.

[16] J. W. Roberts, A non-locally convex F'-space with the Hahn-Banach approximation properly, Proc. Kent State Conference, Springer Lecture Notes 604 (1977).

[17] S. Rolewicz, Metric linear spaces, Warsaw 1972.

[18] L. Schwartz, Les applications 0-radonifiantes dans les espaces de suite, Seminairo Laurent Schwartz Applications Radonifiantes 1964-1970, Exposé No. 26.

[19] P. Turpin, Oonvexités dans les espaces vectoriels topologiques généraux, Diss. Math. 131 (1976).

[20] E. M. Stein and N. J. Woiss, On the convergence of Poisson integrats, Trans. Amer. Math. Soc. 14:0 (1969), 35-54.

Received June 29, 1978 